\title{
MINIMIZERS FOR A DOUBLE-WELL PROBLEM WITH AFFINE BOUNDARY CONDITIONS
}

\author{
Grégoire ALLAIRE, Véronique LODS \\ Laboratoire d'Analyse Numérique \\ Université Paris 6 \\ 75252 Paris Cedex 05, France
}

\begin{abstract}
This paper is concerned with the existence of minimizers for functionals having a double-well integrand with affine boundary conditions. Such functionals are related to the so-called Kohn-Strang functional which arises in optimal shape design problems in electrostatics or elasticity. They are known to be not quasi-convex, and therefore existence of minimizers is, in general, guaranteed only for their quasi-convex envelopes. We generalize previous results in [1] and give necessary and sufficient conditions on the affine boundary conditions for existence of minimizers. Our method relies on the computation of the quasiconvexification of these functionals by using homogenization theory. We also prove by a general argument that their rank-one convexifications coincide with their quasi-convexifications.
\end{abstract}

Key words: homogenization, quasiconvexity, rank-one convexity, calculus of variations, relaxation, optimal design.

\section{Introduction}

Let $\Omega$ be a bounded domain of $\mathbb{R}^{n}$. Let $\xi$ be a constant matrix in $\mathbb{R}^{n N}$, which has $N$ lines and $n$ columns. Let $D_{\xi}$ be the following space of vector-valued functions with affine boundary data

$$
D_{\xi}=\left\{\xi \cdot x+H_{0}^{1}\left(\Omega ; \mathbb{R}^{N}\right)\right\} .
$$

The derivatives of a vector-valued function $u(x)$ in $D_{\xi}$ are denoted by $D u=$ $\left(\partial u_{i} / \partial x_{j}\right) \in \mathbb{R}^{n N}$. This paper is devoted to the question of existence of minimizers in $D_{\xi}$ for the following functional

$$
F(u)=\int_{\Omega} f(D u) d x,
$$

where the integrand $f$ is a function from $\mathbb{R}^{n N}$ into $\mathbb{R}^{+}$, defined by

$$
f(\eta)=\min \left(\lambda+\alpha|\eta|^{2}, \beta|\eta|^{2}\right)
$$


with $0<\alpha<\beta<+\infty, 0<\lambda<+\infty$, and $|\eta|$ is the Frobenius norm of the $N \times n$ matrix $\eta$. When $\beta=+\infty$, the functional (1-2) is the so-called KohnStrang functional. In dimension $n=2$, it was introduced by Kohn and Strang in [18] as a model problem in the field of shape optimization. When $\beta<+\infty$, the minimization problem associated to (1-2) can still be shown to be equivalent to an optimal design problem in electrostatics. However, in this latter case it is also equivalent to a double-well energy minimization problem.

It is by now well-known that the functional $F$ is not (sequentially) weakly lower semi-continuous on $D_{\xi}$. Therefore, the direct method of the calculus of variations does not yield the existence of minimizers for (1) in $D_{\xi}$. Rather, one needs to introduce the relaxed functional (see [7])

$$
\bar{F}(u)=\int_{\Omega} Q f(D u) d x,
$$

where $Q f$ is the quasiconvex envelope of $f$ defined by

$$
Q f(\eta)=\inf _{\varphi \in H_{0}^{1}\left(Y ; \mathbb{R}^{N}\right)} \int_{Y} f(\eta+D \varphi) d y,
$$

where $Y=(0,1)^{n}$ is the unit cube of $\mathbb{R}^{n}$. Then, $u_{0}(x)=\xi x$ is a minimizer of the relaxed functional $\bar{F}$ on $D_{\xi}$, and

$$
Q f(\xi)=\inf _{u \in D_{\xi}} \frac{1}{|\Omega|} \int_{\Omega} f(D u) d x .
$$

When $\beta=+\infty$, the function $f$ simplifies in

$$
f(\eta)= \begin{cases}\lambda+\alpha|\eta|^{2}, & \eta \neq 0, \\ 0, & \eta=0,\end{cases}
$$

and the quasiconvexification $Q f$ has been explicitly computed in [1]. Furthermore, the question of finding conditions for existence or non-existence of minimizers in $D_{\xi}$ of (1) has also been addressed in this paper (previous results were already obtained in the two-dimensional case in [8], [18]).

The first result of [1] gives the value of the quasiconvexification $Q f$ (which turns out to coincides with the rank-one convexification $R f$ ) : denoting by $\eta_{1}, \ldots, \eta_{n}$ the square roots of the eigenvalues of $\eta^{t} \eta$,

$$
Q f(\eta)= \begin{cases}\alpha|\eta|^{2}+\lambda & \text { if } \sum_{i=1}^{n} \eta_{i} \geq \sqrt{\frac{\lambda}{\alpha}} \\ \alpha|\eta|^{2}-\alpha\left(\sum_{i=1}^{n} \eta_{i}\right)^{2}+2 \sqrt{\lambda \alpha} \sum_{i=1}^{n} \eta_{i} & \text { if } \sum_{i=1}^{n} \eta_{i}<\sqrt{\frac{\lambda}{\alpha}} .\end{cases}
$$

The next result of [1] is the following

Theorem 1.1 The minimization of (1) over $D_{\xi}$ admits the trivial minimizer $u(x)=\xi x$ when $\xi$ is such that $f(\xi)=Q f(\xi)$. When $f(\xi)>Q f(\xi)$, then there 
exists a minimizer if rank $\xi=n$, while there is no minimizer if rank $\xi=1$, and in the remaining case, $2 \leq \operatorname{rank} \xi \leq n-1$, there is no minimizer $u(x)$ such that the distance between the boundary $\partial \Omega$ and the set $Z_{u}=\{x \in \Omega \mid \nabla u(x)=0\}$ is strictly positive.

Remark that the above theorem of [1] does not furnish a necessary and sufficient condition for existence of minimizers, since in the case $f(\xi)>Q f(\xi)$ and $2 \leq \operatorname{rank} \xi \leq n-1$ there is a technical condition on the minimizer for its non-existence. Such a restrictive condition is due to technical difficulties caused by the degeneracy of $f$ when $\beta=+\infty$. Remark that, on the other hand, the assumption $\beta=+\infty$ greatly simplifies the algebra in the computation of $Q f$. It was conjectured in [1] that this technical condition is unnecessary for proving non-existence in the case $f(\xi)>Q f(\xi)$ and $2 \leq \operatorname{rank} \xi \leq n-1$.

The goal of the present paper is to generalize the results of [1] in two directions. Firstly, we extend Theorem 1.1 to the case $\beta<+\infty$ and we remove the technical condition on $Z_{u}$. In other words, we give necessary and sufficient conditions for the existence of minimizers of (1) over $D_{\xi}$ (see Theorem 3.9). When $\beta<+\infty$, the algebra is much more involved than in [1] (for example, the explicit expression of $Q f$ in Theorem 3.7 is very complicated), so our proofs rely more on general arguments rather than on explicit computations. Secondly, we also extend our results to the linearized elasticity setting. More precisely, when $N=n$, we replace the gradient $D u$ by the strain tensor $e(u)=1 / 2\left(\partial u_{i} / \partial x_{j}+\partial u_{j} / \partial x_{i}\right)$, and, for a symmetric matrix $\xi$, we consider the following minimization

$$
\inf _{u \in D_{\xi}} F(u)=\int_{\Omega} f(e(u)) d x
$$

where the integrand $f$ is defined from $\mathbb{R}^{n^{2}}$ into $\mathbb{R}^{+}$by

$$
f(\eta)=\min (\lambda+A \eta \cdot \eta, B \eta \cdot \eta)
$$

with $A$ and $B$ two isotropic positive definite fourth-order tensors defined by $A \eta=2 \mu_{A} \eta+\left(\kappa_{A}-2 \mu_{A} / n\right) \operatorname{tr}(\eta) I_{2}$ and $B \eta=2 \mu_{B} \eta+\left(\kappa_{B}-2 \mu_{B} / n\right) \operatorname{tr}(\eta) I_{2}$. For such a functional, we also give necessary and sufficient conditions for the existence of minimizers of (5) (see Theorem 5.6). Actually the sufficient condition of existence for (5) was already obtained in [15], [16]. Finally, as a byproduct of our analysis, we found a new and systematic proof of the equality between the quasiconvexification and the rank-one convexification for such types of functionals (see Proposition 3.5). This latter property has also been obtained independently by Milton [22].

Our motivation for studying such a problem is twofold. First, there are many attempts to provide an existence theory in the calculus of variations without lower semi-continuity of the functional (i.e. without using the direct method of the calculus of variations). A recent article of Dacorogna and Marcellini [8] has investigated this question for functionals similar to (1) which are not 
quasiconvex. This theory, although very general, does not provide necessary and sufficient conditions for existence of minimizers, even with affine boundary conditions. Our goal is therefore to furnish an explicit example with such conditions, which can be used as a "benchmark" for general theories. We hast to add that our method is very specific to the functional considered here, and can not be extended to other problems (nevertheless, for some non-linear problems or dual energy problems, some generalizations may be found in [1]). Indeed, the key argument is the link between (1-2) and the homogenization theory for two-phase composite materials. In truth, the existence of possible minimizers for (1) or (5) is not merely a question of purely theoretical interest. It also has important consequences in the context of optimal design or phase transitions (see Section 2 below for a brief exploration of these connections). Our second motivation is therefore to provide explicit minimizers of (1) or (5) which can be interpreted as "classical" optimal designs, as opposed to the usual "relaxed" or homogenized optimal designs for this problem.

Let us conclude this introduction by an outline of our paper. Section 2 is dedicated to the link between (1-2) and problems in optimal shape design or phase transitions. Our main results for the minimization problem (1-2) are then stated in Section 3, while their proof are given in Section 4. Finally, Section 5 deals with problem (5) in the linearized elasticity setting.

\section{Link with the double-well problem and opti- mal design.}

We begin by briefly exploring the connection between the energy (2) and optimal design (see Section 4 in [18] for more details). Let us consider two materials of isotropic conductivity $\alpha$ and $\beta$, with $0<\alpha<\beta$, which fill up the domain $\Omega$. We denote by $\chi(x)$ the characteristic function of the subdomain occupied by the conductor $\alpha$. For such an arrangement of the two phases, we compute the solutions $\left(u_{i}\right)_{1 \leq i \leq N}$ of $N$ independent conductivity problems with affine boundary conditions $\bar{\xi}_{i} \cdot x$, where $\left(\xi_{i}\right)_{1 \leq i \leq N}$ are the lines of a matrix $\xi$. Denoting by $u$ the vector-valued function of components $u_{i}$, it is the unique minimizer of

$$
E(\chi)=\min _{u \in D_{\xi}} \int_{\Omega}(\alpha \chi(x)+\beta(1-\chi(x)))|\nabla u(x)|^{2} d x,
$$

where $D_{\xi}$ is the subset of $H^{1}\left(\Omega ; \mathbb{R}^{N}\right)$ made of functions with boundary data $\xi x$. The energy $E(\chi)$ is a global measure of the overall conductivity of the mixture of $\alpha$ and $\beta$ in $\Omega$. Assuming that $\alpha$ is more expensive than $\beta$, we denote by $\lambda>0$ the increment of price to pay for $\alpha$ by unit volume. Seeking the best distribution of materials which is of minimal price and lowest conductivity 
yields the following optimal design problem

$$
\inf _{\chi(x) \in L^{\infty}(\Omega ;\{0,1\})}\left\{E(\chi)+\lambda \int_{\Omega} \chi(x) d x\right\} .
$$

In fact, the true problem of physical interest is to find the mixture of $\alpha$ and $\beta$ with highest overall conductivity when $\beta$ is more expensive than $\alpha$. However, this latter problem fits in our framework only when using a dual formulation of (6), which slightly complicates the exposition (see [18]). Therefore, here we only consider problem (7), although our arguments could easily be generalized to a more realistic optimal design problem (see [1]).

Lemma 2.1 The minimization problem

$$
\inf _{u \in D_{\xi}}\left\{F(u)=\int_{\Omega} \min \left(\beta|\nabla u(x)|^{2}, \alpha|\nabla u(x)|^{2}+\lambda\right) d x\right\}
$$

is equivalent to the optimal design problem (7) in the sense that, if there exists a minimizer $u$ of (8), then the characteristic function $\chi(x)$ of the set where $\alpha|\nabla u(x)|^{2}+\lambda<\beta|\nabla u(x)|^{2}$ is a minimizer of (7), and reciprocally, if there exists a minimizer $\chi$ of (7), then the associated minimizer $u$ of (6) is also a minimizer of (8).

Remark 2.2 Without further constraints on the geometry of the mixture of the two phases, the optimal design problem (7) is known to have no solutions in general (cf. the seminal counter-examples of Murat [24] and Tartar [26]). To obtain a well-posed problem, the original problem (7) must be relaxed through the introduction, as admissible designs, of composite materials that mimic the behavior of minimizing sequences of characteristic functions. Nevertheless, there could exist boundary conditions, corresponding to a special choice of $\xi$, for which a "classical" optimal design (i.e. a true characteristic function) is attainable. Theorem 3.9 provide conditions on $\xi$ that permit to assert or to rule out the existence of such an optimal design.

Proof. We rewrite the optimal design problem as a double minimization

$$
\inf _{\chi(x) \in L^{\infty}(\Omega ;\{0,1\})} \min _{u \in D_{\xi}} \int_{\Omega}\left[(\alpha \chi(x)+\beta(1-\chi(x)))|\nabla u(x)|^{2}+\lambda \chi(x)\right] d x .
$$

The order of minimization is irrelevant. Minimizing in $\chi$ first yields exactly (8). The equivalence between (8) and (7) is therefore obvious.

We now explain the connection with a so-called double-well problem. Minimization of a double-well energy arises in the context of phase transitions in 
material science (see e.g. [4], [17]). Although the true context is that of nonlinear elasticity, one may consider as a primitive model problem the following minimization

$$
\inf _{v \in H_{0}^{1}\left(\Omega ; \mathbb{R}^{N}\right)}\left\{G(v)=\int_{\Omega} \min \left(\beta\left|\nabla v(x)-\xi_{\beta}\right|^{2}, \alpha\left|\nabla v(x)-\xi_{\alpha}\right|^{2}\right) d x\right\}
$$

where $\xi_{\alpha}, \xi_{\beta}$ are two different matrices in $\mathbb{R}^{N n}$. Remark that the quadratic wells do not have the same shape since we still assume that $0<\alpha<\beta$. The following result is well-known among experts (see e.g. section 6 in [16], or [10], [17]) and we reproduce it for the sake of completeness.

Lemma 2.3 Given matrices $\xi_{\alpha}, \xi_{\beta}$, we define a matrix $\xi$ by

$$
\xi=\frac{\beta \xi_{\beta}-\alpha \xi_{\alpha}}{\beta-\alpha}
$$

and a positive constant $\lambda$ by

$$
\lambda=\frac{\alpha \beta}{\beta-\alpha}\left|\xi_{\alpha}-\xi_{\beta}\right|^{2}
$$

Then, the double-well problem (9) is equivalent to the minimization problem

$$
\inf _{u \in D_{\xi}}\left\{F(u)=\int_{\Omega} \min \left(\beta|\nabla u(x)|^{2}, \alpha|\nabla u(x)|^{2}+\lambda\right) d x\right\}
$$

in the sense that, if there exists a minimizer $u$ of (10), then $v(x)=u(x)-\xi x$ is a minimizer of (9), and reciprocally, if there exists a minimizer $v(x)$ of (9), then $u(x)=v(x)+\xi x$ is also a minimizer of (10).

Similarly, given a matrix $\xi$ and a positive constant $\lambda$, there exist two matrices $\xi_{\alpha}, \xi_{\beta}$ (non-unique) such that (9) and (10) are equivalent.

Proof. Given $\xi_{\alpha}, \xi_{\beta}$, we define $\xi$ and $\lambda$ as required. For any $v \in H_{0}^{1}\left(\Omega ; \mathbb{R}^{N}\right)$, $u(x)=v(x)+\xi x$ belongs to $D_{\xi}$. We compute $G(v)$ in terms of $u$

$$
\begin{array}{r}
G(v)=\int_{\Omega} \min \left(\beta|\nabla u|^{2}+2 \beta \nabla u \cdot\left(\xi_{\beta}-\xi\right)+\beta\left|\xi_{\beta}-\xi\right|^{2},\right. \\
\left.\alpha|\nabla u|^{2}+2 \alpha \nabla u \cdot\left(\xi_{\alpha}-\xi\right)+\alpha\left|\xi_{\alpha}-\xi\right|^{2}\right) d x .
\end{array}
$$

The definition of $\xi$ implies that

$$
\xi=\xi_{\alpha}+\frac{\beta}{\beta-\alpha}\left(\xi_{\beta}-\xi_{\alpha}\right)=\xi_{\beta}+\frac{\alpha}{\beta-\alpha}\left(\xi_{\beta}-\xi_{\alpha}\right),
$$

and since $\int_{\Omega}\left(\xi_{\beta}-\xi_{\alpha}\right) \cdot \nabla u d x$ is a null-lagrangian (i.e. is constant for any $u \in D_{\xi}$ ), we obtain

$$
G(v)=F(u)+\beta\left(|\xi|^{2}-\left|\xi_{\beta}\right|^{2}\right)|\Omega|
$$


This proves the desired equivalence of the minimization problems (10) and (9).

Similarly, for given $\xi$ and $\lambda$, and for any unit matrix $\xi^{\prime}$ such that $\xi \cdot \xi^{\prime}=0$ and $\left|\xi^{\prime}\right|=1$, one can find two constants $a$ and $b$ such that

$$
\xi_{\alpha}=\xi+a \xi^{\prime}, \quad \xi_{\beta}=\xi+a \frac{\alpha}{\beta} \xi^{\prime}
$$

and $F(u)=G(v)+b$.

\section{Main results.}

The starting point of our analysis is a well-known lemma concerning the quasiconvexification of $f$. Since its proof is elementary and enlightening, we briefly sketch it.

Lemma 3.1 The quasiconvexification of $f$, defined by (2), is

$$
Q f(\eta)=\inf _{0 \leq \theta \leq 1} \inf _{A \in G_{\theta}}\left\{A \eta^{t} \cdot \eta+\lambda \theta\right\},
$$

where $G_{\theta}$ is the set of all symmetric $n \times n$ matrices which are obtained by the periodic homogenization of a mixture of $\alpha$ and $\beta$ in proportions $\theta$ and $1-\theta$.

Proof. The quasiconvexification of $f$ is defined by

$$
Q f(\eta)=\inf _{\varphi \in H_{\#}^{1}\left(Y ; \mathbb{R}^{N}\right)} \int_{Y} f(\eta+D \varphi) d y
$$

In (12) $Y=(0,1)^{n}$ is a unit cube in $\mathbb{R}^{n}$ and $H_{\#}^{1}\left(Y ; \mathbb{R}^{N}\right)$ denotes the subspace of $H^{1}\left(Y ; \mathbb{R}^{N}\right)$ of periodic functions. Note that the usual definition of the quasiconvexification involves Dirichlet rather than periodic boundary condition for the trial fields (see e.g. [5], [7]). Nevertheless both definitions are easily shown to be equivalent, at least when $f$ is non negative, continuous and grows at most quadratically.

Since $f$ is defined as a minimum, a simple switch in the minimizations leads to

$$
Q f(\eta)=\inf _{\chi \in L^{\infty}(Y ;\{0,1\})}\left\{A_{\chi} \eta^{t} \cdot \eta+\lambda \int_{Y} \chi d y\right\}
$$

where, denoting by $\left(e_{i}\right)_{1 \leq i \leq n}$ the canonical basis of $\mathbb{R}^{n}, A_{\chi}$ is a $n \times n$ symmetric matrix defined by its entries

$$
A_{\chi} e_{i} \cdot e_{j}=\min _{\varphi \in H_{\#}^{1}(Y ; \mathbb{R})} \int_{Y}(\chi \alpha+(1-\chi) \beta)\left(e_{i}+D \varphi\right) \cdot\left(e_{j}+D \varphi\right) d y .
$$


This is precisely the definition of an homogenized matrix obtained by periodic homogenization of a mixture of $\alpha$ and $\beta$ distributed according to the characteristic function $\chi$ (see e.g. [6]). Denoting by $\theta$ the average of $\chi$ in $Y$, we have

$$
Q f(\eta)=\inf _{0 \leq \theta \leq 1}\left\{\inf _{\substack{x \in L^{\infty}(Y ;\{0,1\}) \\ \int_{Y} \chi(y) d y=\theta}} A_{\chi} \eta^{t} \cdot \eta+\lambda \theta\right\} .
$$

For a given $\theta \in[0,1]$, we denote by $G_{\theta}$ the set of all possible homogenized conductivity tensors $A_{\chi}$. Therefore, (14) is the desired result.

Remark 3.2 In the present setting of conductivity with two isotropic phases, the set $G_{\theta}$ is known ([19], [20], [27]). This will allow us to perform an explicit minimization with respect to $A_{\chi}$ (see Proposition 3.3 below). In the context of elasticity (see Section 5), the equivalent of $G_{\theta}$ is unknown. However, it is still possible to compute the minimum of $A_{\chi} \eta^{t} \cdot \eta$ over $G_{\theta}$. The minimal value is called a Hashin-Shtrikman bound on the energy (see Section 5).

Our first result is a simpler formula for the quasiconvex envelope $Q f$. This formula is not yet completely explicit, but rather gives $Q f$ as the minimum value of a convex function of $\theta$. Although this last minimization can be achieved (at the price of a tedious computation), it turns out that the formula of Proposition 3.3 is simpler to use in the sequel.

Proposition 3.3 Let $0 \leq \eta_{1} \leq \cdots \leq \eta_{n}$ be the singular values of the matrix $\eta$. The quasiconvexification of $f$, defined by (2), is

$$
Q f(\eta)=\min _{0 \leq \theta \leq 1}\{f(\theta, \eta)+\lambda \theta\}
$$

where $f(\theta, \eta)$ is a strictly convex function of $\theta$ given by

$$
f(\theta, \eta)=\alpha|\eta|^{2}+\left(a^{+}(\theta)-\alpha\right)\left(\frac{\left(\sum_{i=q}^{n} \eta_{i}\right)^{2}}{\frac{a^{+}(\theta)-\alpha}{a^{-}(\theta)-\alpha}+n-q}+\sum_{i=1}^{q-1} \eta_{i}^{2}\right)
$$

where $a^{+}(\theta)=\theta \alpha+(1-\theta) \beta, a^{-}(\theta)=\left(\theta \alpha^{-1}+(1-\theta) \beta^{-1}\right)^{-1}$, and $q \in\{1, \ldots, n\}$ is the smallest integer such that

$$
\eta_{q}>\eta_{q}^{*}:=\frac{\sum_{i=q}^{n} \eta_{i}}{\frac{a^{+}(\theta)-\alpha}{a^{-}(\theta)-\alpha}+n-q} .
$$

Remark 3.4 The function $f(\theta, \eta)$ is called a Hashin-Shtrikman bound : it is precisely equal to the minimal value of $A \eta^{t} \cdot \eta$, when $A$ runs in the set $G_{\theta}$. When $\eta \neq 0$ and $\theta \neq 0,1$, there always exists a smallest integer $q$ since inequality 
(17) is satisfied for $q=n$. When $\theta=1$, the choice of $q$ is irrelevant and $f(1, \eta)=\alpha|\eta|^{2}$. When $\theta=0$, the smallest integer $q$ to satisfy (17) is $q=n+1$ and $f(0, \eta)=\beta|\eta|^{2}$. When $\eta=0$, then $q=n+1$ and $f(\theta, 0)=0$.

When $\beta=+\infty$, the smallest integer $q$ is easily seen to be such that $n+1-q$ is the rank of $\eta$ (or its number of non-zero singular values). When $\beta<+\infty$, the integer $q$ can be thought of as a measure of the deviation of $\eta$ from an orthogonal matrix : the smallest $q$, the more the singular values of $\eta$ are clustered (in the limit when $\beta-\alpha$ goes to $0, \eta^{t} \eta$ is proportional to the identity if $q=1$ ).

For two anisotropic well-ordered phases, a formula for $f(\theta, \eta)$ has been derived in [15] for the case $q=1$.

The rank-one convex envelope $R f$ of a function $f$ is defined as the largest rank-one convex function which stays below $f$. Recall that a function $g$, defined from $\mathbb{R}^{N \times n}$ into $\mathbb{R}$, is said to be rank-one convex if, for any $t \in[0,1]$ and any matrices $\eta, \zeta$ such that the rank of $(\eta-\zeta)$ is 1 , it satisfies

$$
g(t \eta+(1-t) \zeta) \leq t g(\eta)+(1-t) g(\zeta) .
$$

Rank-one convexity is a weaker property than quasiconvexity, since a quasiconvex function is always rank-one convex (see e.g. [7]). Consequently, $Q f \leq$ $R f \leq f$ for all functions $f$. Actually, rank-one convexity does not imply any kind of lower semicontinuity, but rather that the associated Euler equations are elliptic (see e.g. [7]). Usually, a rank-one convex envelope is much simpler to compute than a quasiconvex envelope. Then, the question arises whether the two coincide. It turns out to be the case in the present setting.

Proposition 3.5 The rank-one convex and quasiconvex envelopes of $f$, defined by (2), are equal, i.e. $Q f=R f$.

Remark 3.6 When $\beta=+\infty$, Proposition 3.5 was proved in [1] by using the explicit formula obtained for $Q f$. Here, we generalize this result thanks to a more general argument which does not require a complete knowledge of $Q f$. Actually, the proof of Proposition 3.5 relies only on Lemma 3.1 and not on Proposition 3.3. This will allow its generalization to the elasticity case. The key ingredient of Proposition 3.5 is the fact that the Hashin-Shtrikman bound $f(\theta, \eta)$ is achieved by sequential laminates. Then, we establish a connection between the lamination formula and the so-called Kohn-Strang algorithm for computing $R f$. This is indeed a very general connection which has also been remarked independently by Milton (see the last chapter in [22]).

From Proposition 3.3 we can compute an explicit formula for the quasiconvexification $Q f$. This formula is not very useful since it is quite cumbersome. In particular, the proof of the next Theorem 3.9 on necessary and sufficient conditions of existence of minimizers does not use this formula, but rather relies merely on Proposition 3.3. 
Theorem 3.7 Let $0 \leq \eta_{1} \leq \cdots \leq \eta_{n}$ be the singular values of the matrix $\eta$. Let $q \in\{1, \ldots, n\}$ be the smallest integer such that

$$
\eta_{q}>\frac{\sum_{i=q}^{n} \eta_{i}}{\frac{\beta}{\alpha}+n-q} .
$$

Define ordered real numbers $c_{n} \geq \cdots \geq c_{q} \geq c_{q-1}$ by

$$
c_{p}=\sum_{i=1}^{p-1} \eta_{i}^{2}+\left(n-p+\frac{\beta}{\alpha}\right) \eta_{p}^{2} \text { for } p \in\{q, \ldots, n\},
$$

and

$$
c_{q-1}=\sum_{i=1}^{q-1} \eta_{i}^{2}+\frac{\left(\sum_{i=q}^{n} \eta_{i}\right)^{2}}{n-q+\frac{\beta}{\alpha}} .
$$

The quasiconvexification of $f$, defined by (2), is given by

1. if $\frac{\lambda}{\beta-\alpha} \geq c_{n}$, then

$$
Q f(\eta)=\beta|\eta|^{2},
$$

2. if, for $p \in\{q, \ldots, n\}, c_{p} \geq \frac{\lambda}{\beta-\alpha} \geq c_{p-1}$, then

$$
\begin{gathered}
Q f(\eta)=\alpha|\eta|^{2}+(\beta-\alpha) \sum_{i=1}^{p-1} \eta_{i}^{2}-\alpha\left(\sum_{i=p}^{n} \eta_{i}\right)^{2} \\
-\alpha(n-p+1)\left(\frac{\lambda}{\beta-\alpha}-\sum_{i=1}^{p-1} \eta_{i}^{2}\right)+2 \alpha \sqrt{n-p+\frac{\beta}{\alpha}} \sqrt{\frac{\lambda}{\beta-\alpha}-\sum_{i=1}^{p-1} \eta_{i}^{2}}\left(\sum_{i=p}^{n} \eta_{i}\right),
\end{gathered}
$$

3. if $c_{q-1} \geq \frac{\lambda}{\beta-\alpha}$, then

$$
Q f(\eta)=\alpha|\eta|^{2}+\lambda .
$$

Remark 3.8 When $\eta \neq 0$, there always exists a smallest integer $q$ since inequality (18) is satisfied for $q=n$. When $\eta=0$, the value of $q$ is irrelevant and $Q f(0)=0$.

Finally, we state the main result of this paper which gives a necessary and sufficient condition for the existence of minimizers for the double-well problem.

Theorem 3.9 Let $0 \leq \xi_{1} \leq \cdots \leq \xi_{n}$ be the singular values of the matrix $\xi$. Let $q \in\{1, \ldots, n\}$ be the smallest integer such that

$$
\xi_{q}>\frac{\sum_{i=q}^{n} \xi_{i}}{\frac{\beta}{\alpha}+n-q} .
$$

The minimization problem (1) admits a minimizer over $D_{\xi}$ if and only if one of the following conditions holds 
1.

$$
\frac{\lambda}{\beta-\alpha} \geq|\xi|^{2}+\frac{\beta-\alpha}{\alpha} \xi_{n}^{2}
$$

2.

$$
\frac{\lambda}{\beta-\alpha} \leq \sum_{i=1}^{q-1} \xi_{i}^{2}+\frac{\left(\sum_{i=q}^{n} \xi_{i}\right)^{2}}{n-q+\frac{\beta}{\alpha}}
$$

3. $q=1$ and

$$
\frac{\left(\sum_{i=1}^{n} \xi_{i}\right)^{2}}{n-1+\frac{\beta}{\alpha}} \leq \frac{\lambda}{\beta-\alpha} \leq\left(n-1+\frac{\beta}{\alpha}\right) \xi_{1}^{2} .
$$

Remark 3.10 Of course, when $\beta=+\infty$ the existence condition of Theorem 3.9 reduces to that of Theorem 1.1, proved in [1]. However, the technical condition involved in Theorem 1.1 can now be removed by using the method of proof of Theorem 5.6 in section 5 (this method relies on the Hashin-Shtrikman variational principle rather than on the knowledge of the $G$-closure as in the proof of Theorem 3.9). For more details when $\beta=+\infty$, we refer to Remarks 5.8 and 5.9 .

\section{Proofs of Theorems 3.7 and 3.9.}

This section is devoted to the computation of the quasiconvexification $Q f$ and to establishing a necessary and sufficient condition of existence for minimizers of (1-2).

Proof of Proposition 3.3. In view of Lemma 3.1, we merely have to compute

$$
f(\theta, \eta)=\inf _{A \in G_{\theta}} A \eta^{t} \cdot \eta
$$

For a mixture of two isotropic conductors $\alpha$ and $\beta$, the set $G_{\theta}$ has been explicitly computed by Tartar [27] (see also [20], [27]). It is the set of all $n \times n$ symmetric matrices with eigenvalues $\lambda_{1}, \ldots, \lambda_{n}$ satisfying

$$
\left\{\begin{array}{l}
a^{-}(\theta)=\left(\frac{\theta}{\alpha}+\frac{1-\theta}{\beta}\right)^{-1} \leq \lambda_{i} \leq a^{+}(\theta)=\theta \alpha+(1-\theta) \beta, \text { for all } 1 \leq i \leq n \\
\sum_{j=1}^{n} \frac{1}{\lambda_{j}-\alpha} \leq S_{\alpha}=\frac{1}{a^{-}(\theta)-\alpha}+\frac{n-1}{a^{+}(\theta)-\alpha} \\
\sum_{j=1}^{n} \frac{1}{\beta-\lambda_{j}} \leq S_{\beta}=\frac{1}{\beta-a^{-}(\theta)}+\frac{n-1}{\beta-a^{+}(\theta)}
\end{array}\right.
$$

We rewrite $A \eta^{t} \cdot \eta=\operatorname{Tr}(A H)$, where $H=\eta^{t} \eta$ is a $n \times n$ symmetric matrix having eigenvalues $0 \leq \eta_{1}^{2} \leq \ldots \leq \eta_{n}^{2}$, with $0 \leq \eta_{1} \leq \ldots \leq \eta_{n}$ the singular values 
of $\eta$. By a result of von Neumann (see e.g. [23]), the minimum is attained when $A$ and $H$ are diagonal in the same basis. Therefore,

$$
f(\theta, \eta)=\min \left\{\sum_{i=1}^{n} \lambda_{i} \eta_{n+1-i}^{2}\right\},
$$

where the minimum is taken on $\lambda_{1} \leq \lambda_{2} \ldots \leq \lambda_{n}$, and the eigenvalues $\lambda_{i}$ belong to the convex set defined by (20). Therefore, existence of a minimizer is obvious. To compute the minimum in (21), we write its associated Euler equation. To simplify the problem, we forget the ordering of the $\left(\lambda_{i}\right)_{i=1, . ., n}$ and we make the following remarks. Let $\left(\lambda_{i}\right)_{i=1, . ., n}$ be a set of eigenvalue belonging to (20). If one of the eigenvalues $\lambda_{i}$ is equal to $a^{-}(\theta)$, then all the other ones are equal to $a^{+}(\theta)$. If the two last inequalities in (20) are saturated, i.e.

$$
\sum_{j=1}^{n} \frac{1}{\lambda_{j}-\alpha}=S_{\alpha}, \text { and } \sum_{j=1}^{n} \frac{1}{\beta-\lambda_{j}}=S_{\beta},
$$

then there exists an index $i_{0} \in\{1, \ldots, n\}$ such that $\lambda_{i_{0}}=a^{-}(\theta)$ and $\lambda_{i}=a^{+}(\theta)$ for all $i \neq i_{0}$ (this can be checked easily by a simple computation). Therefore, if the minimizer of (21) is not such a set of eigenvalues, the only constraints in (20) that can be saturated are $\lambda_{i} \leq a^{+}(\theta)$ and $\sum_{j=1}^{n} \frac{1}{\lambda_{j}-\alpha} \leq S_{\alpha}$. In this case, the Euler equations are

$$
\eta_{n+1-i}^{2}=\frac{C}{\left(\lambda_{i}-\alpha\right)^{2}}-D_{i}
$$

for all $1 \leq i \leq n$, with $C$ and $D_{i}$ positive constants such that

$$
C=0 \text { if } \sum_{j=1}^{n} \frac{1}{\lambda_{j}-\alpha}<S_{\alpha}, \text { and } D_{i}=0 \text { if } \lambda_{i}<a^{+}(\theta) .
$$

Let us define two subsets $I$ and $J$, such that $I \cup J=\{1, . ., n\}$, by

$$
I=\left\{i \in\{1, . ., n\}, \lambda_{i}<a^{+}(\theta)\right\}, J=\left\{i \in\{1, . ., n\}, \lambda_{i}=a^{+}(\theta)\right\} .
$$

Thus

$$
\eta_{n+1-i}^{2}=\frac{C}{\left(\lambda_{i}-\alpha\right)^{2}}-D_{i}
$$

Since $D_{i}=0$ for $i \in I$, it implies that $\eta_{n+1-j}<\eta_{n+1-i}$ for all $j \in J, i \in I$. Consequently, there exists $q \in\{1, . ., n\}$ such that

$$
I=\{1, . ., n+1-q\}, J=\{n+2-q, . ., n\} .
$$

From the equality $S_{\alpha}=\sum_{j=1}^{n} \frac{1}{\lambda_{j}-\alpha}$, we obtain

$$
\sqrt{C}=\frac{\sum_{i=q}^{n} \eta_{i}}{\frac{n-q}{a^{+}(\theta)-\alpha}+\frac{1}{a^{-}(\theta)-\alpha}},
$$


and, for all $i \in I$,

$$
\lambda_{i}=\alpha+\frac{\sqrt{C}}{\eta_{n+1-i}} .
$$

This yields the following value of $f(\theta, \eta)$

$$
f(\theta, \eta)=\alpha|\eta|^{2}+\left(a^{+}(\theta)-\alpha\right)\left(\frac{\left(\sum_{i=q}^{n} \eta_{i}\right)^{2}}{\frac{a^{+}(\theta)-\alpha}{a^{-}(\theta)-\alpha}+n-q}+\sum_{i=1}^{q-1} \eta_{i}^{2}\right) .
$$

It is easily checked that $\lambda_{1} \leq \ldots \leq \lambda_{n}, S_{\beta}>\sum_{j=1}^{n} \frac{1}{\beta-\lambda_{j}}$, and that $\lambda_{i} \leq \lambda_{n+1-q}<$ $a^{+}(\theta)$ for all $i \in I$ if and only if

$$
\eta_{q}>\eta_{q}^{*}:=\frac{\sum_{i=q}^{n} \eta_{i}}{\frac{a^{+}(\theta)-\alpha}{a^{-}(\theta)-\alpha}+n-q} .
$$

Equivalently, $q$ is the smallest integer to satisfy this property. When $q<n$, we check that $a^{-}(\theta)<\lambda_{1}$. When $q=n$, we obtain $\lambda_{1}=a^{-}(\theta)$ and $\lambda_{i}=a^{+}(\theta)$ for $i \geq 2$.

To finish the proof it remains to check that $f(\theta, \eta)$ is strictly convex in $\theta$. For a fixed integer $p \in\{1, \ldots, n\}$, we define a function $f_{p}(\theta, \eta)$ by

$$
f_{p}(\theta, \eta)=\alpha|\eta|^{2}+\left(a^{+}(\theta)-\alpha\right)\left(\frac{\left(\sum_{i=p}^{n} \eta_{i}\right)^{2}}{\frac{a^{+}(\theta)-\alpha}{a^{-}(\theta)-\alpha}+n-p}+\sum_{i=1}^{p-1} \eta_{i}^{2}\right) .
$$

For $\eta_{p} \neq 0$, we also define a number $\theta_{p}$ such that $\eta_{p}=\eta_{p}^{*}\left(\theta_{p}\right)$, i.e.

$$
\theta_{p}=\frac{\alpha}{\beta-\alpha}\left(\frac{\sum_{i=p}^{n} \eta_{i}}{\eta_{p}}-(n-p+1)\right) .
$$

Remark that $\theta_{n}=0$ and $\theta_{p+1} \leq \theta_{p}$. With the convention $\theta_{p}=+\infty$ if $\eta_{p}=0$, and $\theta_{0}=+\infty$, there exists a smallest integer $\tilde{q} \in\{1, \ldots, n\}$ such that

$$
\theta_{\tilde{q}}<1 \leq \theta_{\tilde{q}-1} .
$$

Remark that $\tilde{q}$ is precisely the smallest integer satisfying (18). Since the condition $\eta_{p}>\eta_{p}^{*}$ is equivalent to $\theta>\theta_{p}$, the function $\theta \rightarrow f(\theta, \eta)$ is defined on $[0,1]$ by

$$
\left\{\begin{array}{l}
f(\theta, \eta)=f_{p}(\theta, \eta) \text { if } \theta \in\left[\theta_{p}, \theta_{p-1}\right] \text { for } n \geq p \geq \tilde{q}+1 \\
f(\theta, \eta)=f_{\tilde{q}}(\theta, \eta) \text { if } \theta \in\left[\theta_{\tilde{q}}, 1\right] .
\end{array}\right.
$$

Clearly, each function $f_{p}(\theta, \eta)$ is strictly convex in $\theta$. Furthermore, it is continuously differentiable with respect to $\theta$, in the sense that $f_{p}\left(\theta_{p}, \eta\right)=f_{p+1}\left(\theta_{p}, \eta\right)$, and $f_{p}^{\prime}\left(\theta_{p}, \eta\right)=f_{p+1}^{\prime}\left(\theta_{p}, \eta\right)$. Therefore, $f(\theta, \eta)$ is strictly convex in $\theta$ over $[0,1]$. 
Remark 4.1 In the course of the proof of Proposition 3.3, we have seen that the minimizer $\left(\lambda_{i}\right)_{1 \leq i \leq n}$ is unique. Furthermore, the integer $q$, defined by (22), is such that, the minimizer $\left(\lambda_{i}\right)_{1 \leq i \leq n}$ has its last $q-1$ components $\left(\lambda_{i}\right)_{n+2-q \leq i \leq n}$ equal to $a^{+}(\theta)$. To prove Proposition 3.3 we have chosen to minimize $A \eta^{t} \cdot \eta$ over the $G$-closure set $G_{\theta}$. Alternatively, we could have computed $f(\theta, \eta)$ by using the well-known Hashin-Shtrikman variational principle.

Proof of Theorem 3.7. We use formulae (23-24) to compute $Q f(\eta)$ given by $\min _{0 \leq \theta \leq 1} f(\theta, \eta)+\lambda \theta$. Since $f(\theta, \eta)$ is strictly convex in $\theta$, there exists a unique minimizer $\theta^{*}$. Let $q$ be the smallest integer satisfying (18) (remark that it was called $\tilde{q}$ in the preceding proof of Proposition 3.3). For each fixed integer $p \in\{q, \ldots, n\}$, we compute a possible minimizer $\theta_{p}^{*}$ of $f_{p}(\theta, \eta)$ as the unique root of $f_{p}^{\prime}(\theta, \eta)=0$. If such a root $\theta_{p}^{*}$ exists, then we check whether it belongs or not to the interval $\left[\theta_{p}, \theta_{p-1}\right]$ if $p>q$, and to $\left[\theta_{q}, 1\right]$ if $p=q$. If there are no minimizers $\theta_{p}^{*}$ in these intervals, then the minimizer is $\theta^{*}=0$, or 1 .

Defining $g_{p}(\theta)=f_{p}(\theta, \eta)+\lambda \theta$, a simple computation shows that, if $\frac{\lambda}{\beta-\alpha}-$ $\sum_{i=1}^{p-1} \eta_{i}^{2} \leq 0$, then $g_{p}^{\prime}(\theta)<0$ for any $\theta$, while, if $\frac{\lambda}{\beta-\alpha}-\sum_{i=1}^{p-1} \eta_{i}^{2}>0$, then $\theta_{p}^{*}$ defined by

$$
\frac{\beta-\alpha}{\alpha} \theta_{p}^{*}+(n-p+1)=\sum_{i=p}^{n} \eta_{i} \sqrt{\frac{n-p+\frac{\beta}{\alpha}}{\frac{\lambda}{\beta-\alpha}-\sum_{i=1}^{p-1} \eta_{i}^{2}}}
$$

is the unique real number such that $g_{p}^{\prime}\left(\theta_{p}^{*}\right)=0$. Another easy computation shows that, for $p \in\{q+1, \ldots, n\}$, the condition $\theta_{p} \leq \theta_{p}^{*} \leq \theta_{p-1}$ is equivalent to

$$
c_{p-1} \leq \frac{\lambda}{\beta-\alpha} \leq c_{p}
$$

with $c_{p}=\sum_{i=1}^{p-1} \eta_{i}^{2}+\left(n-p+\frac{\beta}{\alpha}\right) \eta_{p}^{2}$. When $p=q$, the condition $\theta_{q} \leq \theta_{q}^{*} \leq 1$ is equivalent to

$$
c_{q-1}=\sum_{i=1}^{q-1} \eta_{i}^{2}+\frac{\left(\sum_{i=q}^{n} \eta_{i}\right)^{2}}{n-q+\frac{\beta}{\alpha}} \leq \frac{\lambda}{\beta-\alpha} \leq c_{q},
$$

Remark that the condition $\frac{\lambda}{\beta-\alpha}-\sum_{i=1}^{p-1} \eta_{i}^{2}>0$ for the existence of $\theta_{p}^{*}$ is automatically satisfied if $\theta_{p} \leq \theta_{p}^{*} \leq \theta_{p-1}$. Finally, computing the value of $g_{p}\left(\theta_{p}^{*}\right)$ gives the value of $Q f(\eta)$.

We now turn to the necessary and sufficient condition of existence of minimizers for (1-2).

Proof of Theorem 3.9. Let $q \in\{1, \ldots, n\}$ be the smallest integer such that

$$
\xi_{q}>\frac{\sum_{i=q}^{n} \xi_{i}}{\frac{\beta}{\alpha}+n-q},
$$


and let $\theta \in[0,1]$ be the unique minimizer of

$$
Q f(\xi)=\min _{0 \leq \theta \leq 1}(f(\theta, \xi)+\lambda \theta) .
$$

From the proof of Theorem 3.7 we know that $\theta=0$ if and only if $\frac{\lambda}{\beta-\alpha} \geq$ $|\xi|^{2}+\frac{\beta-\alpha}{\alpha} \xi_{n}^{2}$. Then, $\theta=0$ implies that $Q f(\xi)=\beta|\xi|^{2}$. On the other hand, $\theta=1$ if and only if $\frac{\lambda}{\beta-\alpha} \leq \sum_{i=1}^{q} \xi_{i}^{2}+\frac{\left(\sum_{i=q}^{n} \xi_{i}\right)^{2}}{n-q+\frac{\beta}{\alpha}}$. Then, $\theta=1$ implies that $Q f(\xi)=\alpha|\xi|^{2}+\lambda$. In both cases, we have $Q f(\xi)=f(\xi)$. Since by definition of $Q f$,

$$
Q f(\xi)=\inf _{\varphi \in H_{0}^{1}\left(\Omega ; \mathbb{R}^{N}\right)} \frac{1}{|\Omega|} \int_{\Omega} f(\xi+D \varphi) d x
$$

the test function $\varphi=0$ achieves the minimum, and $u(x)=\xi x$ is a minimizer in $D_{\xi}$ for (1-2).

In the remaining case

$$
\sum_{i=1}^{q-1} \xi_{i}^{2}+\frac{\left(\sum_{i=q}^{n} \xi_{i}\right)^{2}}{n-q+\frac{\beta}{\alpha}}<\frac{\lambda}{\beta-\alpha}<|\xi|^{2}+\frac{\beta-\alpha}{\alpha} \xi_{n}^{2},
$$

the minimizer $\theta$ satisfies $0<\theta<1$ and $Q f(\xi)<f(\xi)$. For such values of $\xi$ and $\theta$, there exists a smallest integer $q_{\theta} \geq q$ satisfying

$$
\xi_{q_{\theta}}>\frac{\sum_{i=q_{\theta}}^{n} \xi_{i}}{\frac{a^{+}(\theta)-\alpha}{a^{-}(\theta)-\alpha}+n-q_{\theta}} .
$$

such that (cf. Proposition 3.3)

$$
f(\theta, \xi)=f_{q_{\theta}}(\theta, \xi) .
$$

Finally, for this choice of $\xi, q_{\theta}, \theta$, we look at the unique minimizer $\left(\lambda_{i}\right)_{1 \leq i \leq n}$ in the set $G_{\theta}$, defined by (20), of formula (21) which gives the value of $f(\theta, \bar{\xi})$

$$
f(\theta, \xi)=\min \left\{\sum_{i=1}^{n} \lambda_{i} \xi_{n+1-i}^{2}\right\} .
$$

Recall that in Remark 4.1 it was pointed out that the integer $q_{\theta}$ is such that the minimizer $\left(\lambda_{i}\right)_{1 \leq i \leq n}$ has its last $q_{\theta}-1$ components $\left(\lambda_{i}\right)_{n+2-q_{\theta} \leq i \leq n}$ equal to the arithmetic mean $a^{+}(\theta)$. In view of the proof of Theorem 3.7, $q_{\theta}=1$ if and only if

$$
\frac{\left(\sum_{i=1}^{n} \xi_{i}\right)^{2}}{n-1+\frac{\beta}{\alpha}} \leq \frac{\lambda}{\beta-\alpha} \leq\left(n-1+\frac{\beta}{\alpha}\right) \xi_{1}^{2} .
$$

Let us show that, under assumption (25), there exists a minimizer of (1-2) if and only if $q_{\theta}=1$. 
When $q_{\theta}=1$, none of the optimal eigenvalues $\lambda_{i}$ are equal to $a^{+}(\theta)$. Therefore, the minimizer $\left(\lambda_{i}\right)_{1 \leq i \leq n}$ are such that

$$
\left\{\begin{array}{l}
a^{-}(\theta)<\lambda_{i}<a^{+}(\theta), 1 \leq i \leq n, \\
\sum_{j=1}^{n}\left(\lambda_{j}-\alpha\right)^{-1}=\left(a^{-}(\theta)-\alpha\right)^{-1}+(n-1)\left(a^{+}(\theta)-\alpha\right)^{-1} .
\end{array}\right.
$$

Under these conditions, a well-known result of Tartar [27] in the theory of homogenization and optimal bounds says that a minimizer of (1-2) is obtained with the so-called confocal ellipsoids construction. We refer to [1] for a detailed explanation of this construction in the present setting.

In truth, the difficulty relies in proving that if $q_{\theta}>1$, then there is no minimizer of problem (1-2). Following an idea of [1], we proceed by contradiction : we assume that there exists a minimizer $u(x) \in D_{\xi}$, and we denote by $Z_{u}$ the following subset of $\Omega$

$$
Z_{u}=\left\{x \in \Omega,|D u(x)|^{2} \leq \frac{\lambda}{\beta-\alpha}\right\},
$$

so that

$$
f(D u)=\beta|D u|^{2} \text { on } Z_{u} \text {, and } f(D u)=\alpha|D u|^{2}+\lambda \text { on } \Omega \backslash Z_{u} .
$$

Let $Y=[0,1]^{N}$ be the unit cube in $\mathbb{R}^{N}$. By Vitali's theorem (assuming that $|\partial \Omega|=0$ ), there exists a countable family $\left(\Omega_{i}\right)_{i \geq 1}$ of disjoint homothetics of $\Omega$, with ratio smaller than 1 and $\Omega_{i} \subset Y$, such that

$$
\left|Y \backslash \cup_{i \geq 1} \Omega_{i}\right|=0 .
$$

Since $Z_{u}$ is a subset of $\Omega$, we associate to the family $\left(\Omega_{i}\right)_{i \geq 1}$ the corresponding family $\left(Z_{u i}\right)_{i \geq 1}$ of the homothetics of $Z_{u}$ in $\Omega_{i}$. Let $\chi(x)$ and $\tilde{\chi}(y)$ be the characteristic functions respectively of $\Omega \backslash Z_{u}$ and $Y \backslash \cup_{i \geq 1} Z_{u i}$. Similarly, let us define in $Y$ a function $\tilde{u}(y)$, which is equal, in each copy $\Omega_{i}$, to a properly rescaled version of $u(x)$. Thanks to the affine boundary condition satisfied by $u(x),(\tilde{u}(y)-\xi y)$ belongs to $H_{\#}^{1}\left(Y ; \mathbb{R}^{N}\right)$.

We introduce the homogenized tensor $A_{\chi}$ associated to $\chi$ defined, for any vector $\zeta \in \mathbb{R}^{n}$, by

$$
A_{\chi} \zeta \cdot \zeta=\min _{\phi \in H_{\#}^{1}(Y)} \int_{Y}(\tilde{\chi}(y) \alpha+(1-\tilde{\chi}(y)) \beta)|\zeta+D \phi(y)|^{2} d y .
$$

The matrix $A_{\chi}$ is obtained by periodic homogenization in the unit cell $Y$ of a mixture of $\alpha$ and $\beta$ distributed according to the characteristic function $\tilde{\chi}$ (see e.g. [6]).

The next step is to show that the eigenvalues of this matrix $A_{\chi}$ are precisely the minimizer $\left(\lambda_{i}\right)_{1 \leq i \leq n} \in G_{\theta}$, of (21) which gives the value of $f(\theta, \xi)$. Remark first, that using $\tilde{u}(y)-\xi y$ as a test function in the definition of $A_{\chi}$ leads to

$$
A_{\chi} \xi^{t} \cdot \xi \leq \frac{1}{|\Omega|} \int_{\Omega}(\chi(x) \alpha+(1-\chi(x)) \beta)|D u(x)|^{2} d x .
$$


Recall that, $u$ being a minimizer, one has

$$
Q f(\xi)=\frac{1}{|\Omega|} \int_{\Omega} f(D u) d x=\frac{1}{|\Omega|} \int_{\Omega}(\chi \alpha+(1-\chi) \beta)|D u|^{2} d x+\lambda \theta_{u},
$$

with $\theta_{u}=\frac{1}{|\Omega|} \int_{\Omega} \chi(x) d x=\int_{Y} \tilde{\chi}(y) d y$. On the one hand, a combination of (27) and (28) yields

$$
A_{\chi} \xi^{t} \cdot \xi+\lambda \theta_{u} \leq Q f(\xi) .
$$

On the other hand, from (21) we deduce

$$
Q f(\xi) \leq f\left(\theta_{u}, \xi\right)+\lambda \theta_{u}
$$

Consequently, equality is achieved in (29), and, by uniqueness of the minimizers, $\theta_{u}=\theta$ and $A_{\chi}$ has exactly the eigenvalues $\left(\lambda_{i}\right)_{1 \leq i \leq n}$.

The last step is to obtain a contradiction by recalling that, since $q_{\theta}>1$, one of the eigenvalues of $A_{\chi}$ is equal to the arithmetic mean, i.e. $\lambda_{n}=a^{+}(\theta)$. Indeed, choosing $\zeta=e_{n}$, an eigenvector associated to the eigenvalue $\lambda_{n}$, in (26), the test function $\phi=0$ leads to

$$
a^{+}(\theta)\left|e_{n}\right|^{2}=A_{\chi} e_{n} \cdot e_{n} \leq \int_{Y}(\tilde{\chi} \alpha+(1-\tilde{\chi}) \beta)\left|e_{n}\right|^{2} d x=a^{+}(\theta)\left|e_{n}\right|^{2} .
$$

Therefore, the test function $\phi=0$ is a minimizer of (26). By using Euler's equation associated to (26), we obtain that

$$
\frac{\partial \tilde{\chi}}{\partial y_{n}}(y)=0 \text { in } Y \text {. }
$$

Of course the same result is true for $\chi$, i.e. $\frac{\partial \chi}{\partial x_{n}}(x)=0$ in $\Omega$, but the conclusion is much stronger for $\tilde{\chi}$ since it is true for any Vitali's recovering of $Y$. To obtain a contradiction, we thus choose a particular Vitali's recovering. Remark that, because $\theta_{u}=\theta$ is different from 0 or 1 , there exists two Lebesgue points $x_{0}, x_{1} \in \Omega$ such that $\chi\left(x_{0}\right)=0$ and $\chi\left(x_{1}\right)=1$. Let $\tau \in \mathbb{R}^{n}$ be a translation vector defined by $\tau=\left(x_{0}-x_{1}\right)-\left(x_{0}-x_{1}\right) \cdot\left(e_{n} /\left|e_{n}\right|\right)$. Consider a Vitali's recovering of $Y$ which features two disjoints copies $\Omega_{0}$ and $\Omega_{1}$ of $\Omega$, having the same size and such that $\Omega_{1}$ is translated from $\Omega_{0}$ by a translation $\tau+t e_{n}$, for some $t \in \mathbb{R}$. This implies that the homothetic of $x_{1}$ in $\Omega_{1}$ and that of $x_{0}$ in $\Omega_{0}$ are aligned with the direction $e_{n}$. Therefore, it contradicts the fact that $\frac{\partial \tilde{\chi}}{\partial y_{n}}(y)=0$, and there is no minimizer in $D_{\xi}$ for $(1-2)$ wen $q_{\theta}>1$.

Our proof of the equality between the quasiconvexification $Q f$ and the rankone convexification $R f$ does not use the precise formula obtained above for $Q f$, but rather relies on the following well-known lemma in homogenization (see e.g. [3], [2] for a proof in the context of elasticity). Let us first recall the definition 
of the subset $L_{\theta}$ of $G_{\theta}$ of the so-called finite rank sequential laminates. It is the set of all homogenized matrices $A$ obtained by mixing $\alpha$ around a core of $\beta$ in proportion $\theta$ and $(1-\theta)$ respectively. Let $p \geq 1$ be the rank of the laminate, $\left(e_{i}\right)_{1 \leq i \leq p}$ be the lamination directions, and $\left(m_{i}\right)_{1 \leq i \leq p}$ be lamination parameters satisfying $0 \leq m_{i} \leq 1$ and $\sum_{i=1}^{p} m_{i}=1$. Then $A \in L_{\theta}$ if

$$
(1-\theta)\left(A-\alpha I_{2}\right)^{-1}=\left(\beta I_{2}-\alpha I_{2}\right)^{-1}+\theta \sum_{i=1}^{p} m_{i} \frac{e_{i} \otimes e_{i}}{\alpha\left|e_{i}\right|^{2}}
$$

Remark that in (30) it is always possible to take $p \leq \frac{n(n+1)}{2}+1$ by Caratheodory theorem since the set of all matrices $\sum_{i=1}^{p} m_{i} e_{i} \otimes e_{i}$ is convex in dimension $n(n+1) / 2$.

Lemma 4.2 The Hashin-Shtrikman bound defined by

$$
f(\theta, \eta)=\inf _{A \in G_{\theta}} A \eta^{t} \cdot \eta
$$

is actually equal to

$$
f(\theta, \eta)=\min _{A \in L_{\theta}} A \eta^{t} \cdot \eta
$$

where the minimum is attained in $L_{\theta}$ by a sequential laminate of rank less than $n$, with lamination directions $\left(e_{j}\right)_{j=1, . ., n}$ which are the eigenvectors of the symmetric matrix $\eta^{t} \eta$.

Proof of Proposition 3.5. Let $\eta$ be a fixed matrix in $\mathbb{R}^{n N}$. By Proposition 3.3 , we know that there exists $\theta^{*} \in[0,1]$, such that

$$
Q f(\eta)=f\left(\theta^{*}, \eta\right)+\lambda \theta^{*}
$$

Besides, Lemma 4.2 implies the existence of a rank- $n$ sequential laminate $A_{n}$ in $L_{\theta}$ such that

$$
f\left(\theta^{*}, \eta\right)=A_{n} \eta^{t} \cdot \eta
$$

and the directions of laminations are the singular vectors $\left(e_{j}\right)_{j=1, . ., n}$ of $\eta$. Without any precise knowledge of the optimal parameters $e_{i}, m_{i}$ allowing to calculate $A_{n}$, we shall prove that $Q f=R f$ by establishing a connection between the iterative lamination process and the iterative algorithm of Kohn and Strang [18] for computing $R f$. The latter algorithm amounts to compute a sequence of functions $\left(f^{k}(\eta)\right)_{k \geq 0}$, defined by

$$
\left\{\begin{array}{l}
f^{0}(\eta)=f(\eta) \\
f^{k+1}(\eta)=\inf \left\{\theta f^{k}\left(\eta^{\prime}\right)+(1-\theta) f^{k}\left(\eta^{\prime \prime}\right)\right\}
\end{array}\right.
$$

where the infimum is taken over all parameters $\theta, \eta^{\prime}, \eta^{\prime \prime}$ such that

$$
0 \leq \theta \leq 1, \eta=\theta \eta^{\prime}+(1-\theta) \eta^{\prime \prime}, \operatorname{rank}\left(\eta^{\prime}-\eta^{\prime \prime}\right) \leq 1
$$


By construction, the sequence $f^{k}$ converges to $R f$ as $k$ goes to $+\infty$. To prove the equality $Q f=R f$, we have only to check that

$$
f^{n}(\eta) \leq Q f(\eta)
$$

Indeed, since the sequence $\left(f^{j}\right)_{1 \leq j \leq n}$ decreases, this inequality implies that

$$
R f(\eta) \leq f^{n}(\eta) \leq Q f(\eta),
$$

and since, by definition, we have $Q f \leq R f$, this implies equality. Moreover, from (33) we will deduce that the sequence $\left(f^{j}(\eta)\right)$ is steady after $j \geq n$.

In the sequel we use the polar decomposition of $\eta$

$$
\eta=\sum_{i=1}^{n} \eta_{i} h_{i} \otimes e_{i}
$$

where $\left(h_{i}\right)_{i=1, \ldots, N}$ and $\left(e_{i}\right)_{i=1, \ldots, N}$ are two orthonormal families in $\mathbb{R}^{N}$, and $0 \leq \eta_{1} \leq . . \leq \eta_{n}$ are the singular values of $\eta$.

The rank- $n$ sequential laminate $A_{n}$, allowing to calculate $f\left(\theta^{*}, \eta\right)$ and defined by (30) with $p=n$ and $\left(e_{i}\right)_{i=1, \ldots, N}$ the singular vectors of $\eta$, can be actually calculated by repeating $n$ times a simple rank-one lamination. More precisely, it is obtained by induction from the sequence of laminates $A_{k}$, which is built at each step by a single lamination of $A:=\alpha I_{2}$ and $A_{(k-1)}$ (with $\left.A_{0}=\beta I_{2}\right)$ in the direction $e_{k}$ and in the respective proportions $\theta_{k}$ and $1-\theta_{k}$

$$
\left(1-\theta_{k}\right)\left(A_{k}-A\right)^{-1}=\left(A_{k-1}-A\right)^{-1}+\theta_{k} f_{A}\left(e_{k}\right),
$$

where

$$
f_{A}\left(e_{k}\right)=\frac{e_{k} \otimes e_{k}}{\alpha\left|e_{k}\right|^{2}} .
$$

The volume fraction of $\beta$ at step $k$, is denoted by $\psi_{k}$, which is given by the formula

$$
\psi_{0}=1, \quad \psi_{k}=\Pi_{i=1}^{k}\left(1-\theta_{i}\right),
$$

so that

$$
\psi_{n}=1-\theta^{*} .
$$

To obtain $Q f=R f$, we prove inequality (33) without calculating $f^{n}(\eta)$. We first check, by induction, the following properties, denoted by $I(k)$,

$$
f^{k}(\tilde{\eta}) \leq A_{k} \tilde{\eta}^{t} \cdot \tilde{\eta}+\lambda\left(1-\psi_{k}\right),
$$

for all matrix $\tilde{\eta} \in \mathbb{R}^{n N}$, having the same singular vectors $\left(h_{j}\right)_{j=1, \ldots, n}$ and $\left(e_{j}\right)_{j=1, \ldots, n}$ than $\eta\left(A_{k}\right.$ is defined by (34) and $\psi_{k}$ by $\left.(35)\right)$. The property $I(0)$ is satisfied since $A_{0}=\beta I_{2}, f^{0}=f$, and $\psi_{0}=1$. Assume that $I(k)$ is satisfied and 
let us check $I(k+1)$. Denoting by $E_{k+1}=h_{k+1} \otimes e_{k+1}$ and $c_{k}$ a constant in $\mathbb{R}$ (to be determined later, see (41)), define

$$
\tilde{\eta}^{\prime}=\tilde{\eta}-\theta_{k+1} c_{k} E_{k+1}
$$

and

$$
\tilde{\eta}^{\prime \prime}=\tilde{\eta}+\left(1-\theta_{k+1}\right) c_{k} E_{k+1} .
$$

We check that the rank of $\left(\tilde{\eta}^{\prime}-\tilde{\eta}^{\prime \prime}\right)$ is less or equal to 1 , and that

$$
\tilde{\eta}=\left(1-\theta_{k+1}\right) \tilde{\eta}^{\prime}+\theta_{k+1} \tilde{\eta}^{\prime \prime}
$$

Therefore, the definition of $f^{k+1}$ and the inequality $f^{k}\left(\eta^{\prime \prime}\right) \leq f\left(\eta^{\prime \prime}\right) \leq\left(A \eta^{\prime \prime}\right.$. $\left.\eta^{\prime \prime}+\lambda\right)$ yield

$$
f^{k+1}(\tilde{\eta}) \leq\left(1-\theta_{k+1}\right) f^{k}\left(\tilde{\eta}^{\prime}\right)+\theta_{k+1}\left(A \eta^{\prime \prime} \cdot \eta^{\prime \prime}+\lambda\right) .
$$

Since the matrices $\tilde{\eta}^{\prime}$ and $\eta$ have the same singular directions, property $I(k)$ implies

$$
f^{k}\left(\tilde{\eta}^{\prime}\right) \leq A_{k} \tilde{\eta}^{\prime t} \cdot \tilde{\eta}^{\prime}+\lambda\left(1-\psi_{k}\right) .
$$

Besides, definition (35) of the sequence $\psi_{k}$ implies that $\left(1-\psi_{k+1}\right)=\theta_{k+1}+$ $\left(1-\theta_{k+1}\right)\left(1-\psi_{k}\right)$, so that (38) and (39) lead to

$$
f^{k+1}(\tilde{\eta}) \leq \lambda\left(1-\psi_{k+1}\right)+\left(1-\theta_{k+1}\right) S\left(c_{k}\right),
$$

with

$$
S\left(c_{k}\right)=A_{k} \tilde{\eta}^{\prime t} \cdot \tilde{\eta}^{\prime}+\frac{\theta_{k+1}}{1-\theta_{k+1}} A \eta^{\prime \prime} \cdot \eta^{\prime \prime}
$$

The quadratic function $S\left(c_{k}\right)$ is minimum for

$$
c_{k}^{*}=\frac{\left(A_{k}-A\right) \tilde{\eta}^{t} \cdot E_{k+1}}{\left(1-\theta_{k+1}\right) A E_{k+1}^{t} \cdot E_{k+1}+\theta_{k+1} A_{k} E_{k+1}^{t} \cdot E_{k+1}},
$$

which simplifies in

$$
c_{k}^{*}=\frac{\left(A_{k}-A\right) \tilde{\eta}^{\prime t} \cdot E_{k+1}}{A E_{k+1}^{t} \cdot E_{k+1}},
$$

and the minimum is

$$
S\left(c_{k}^{*}\right)=\left(A_{k}-A\right) \tilde{\eta}^{\prime t} \cdot\left(\tilde{\eta}+\theta_{k+1} c_{k}^{*} E_{k+1}\right)-\left(c_{k}^{*}\right)^{2} \theta_{k+1} A E_{k+1}^{t} \cdot E_{k+1}+\frac{A \tilde{\eta}^{t} \cdot \tilde{\eta}}{1-\theta_{k+1}} .
$$

Inequality (40) implies property $I(k+1)$ if we check the following inequality

$$
S\left(c_{k}^{*}\right) \leq \frac{A_{k+1} \tilde{\eta}^{t} \cdot \tilde{\eta}}{1-\theta_{k+1}} .
$$


From the single lamination formula (34), we can compute exactly the $n \times n$ matrix $A_{k+1}$ (which is diagonal in the basis $\left(e_{i}\right)_{i=1, \ldots, N}$ of the singular vectors of $\eta$ ), but we prefer to avoid this calculation which cannot be done in the elasticity case (cf. Proposition 5.4 in section 5). Rather, by using the Legendre transform, we rewrite

$$
\left(1-\theta_{k+1}\right)^{-1}\left(A_{k+1}-A\right) \tilde{\eta}^{t} \cdot \tilde{\eta}=\max _{\xi \in \mathbb{R}^{n^{2}}} h(\xi)
$$

where

$$
h(\xi)=2 \xi^{t} \cdot \tilde{\eta}-\left(A_{k}-A\right)^{-1} \xi^{t} \cdot \xi-\theta_{k+1} f_{A}\left(e_{k+1}\right) \xi^{t} \cdot \xi .
$$

For $\xi_{k}^{t}=\left(A_{k}-A\right)\left(\tilde{\eta}^{\prime}\right)^{t}$, we obtain a lower bound

$$
\left(1-\theta_{k+1}\right)^{-1}\left(A_{k+1}-A\right) \tilde{\eta}^{t} \cdot \tilde{\eta} \geq h\left(\xi_{k}\right) .
$$

From (43) and (45) we remark that

$$
S\left(c_{k}^{*}\right)=h\left(\xi_{k}\right)+\frac{A \tilde{\eta}^{t} \cdot \tilde{\eta}}{1-\theta_{k+1}},
$$

and consequently (46) is the desired inequality (44). Therefore, property $I(n)$ is established by induction, and this proves that $f^{n}(\eta) \leq Q f(\eta)$.

Remark 4.3 The inequality (46) is in fact an equality because we can check that the matrix $\xi_{k}$ is a minimum of the function $h$ defined in (45).

\section{Generalization to the elasticity case.}

This section is devoted to the generalization of Section 3 for a double-well energy minimization in the context of linearized elasticity. As before, $\Omega$ is a bounded domain in $\mathbb{R}^{n}$. From now on, $u(x)$ denotes a vector-valued function from $\Omega$ into $\mathbb{R}^{n}$ (i.e. $N=n$ ). Let $\xi$ be a symmetric $n \times n$ matrix. We define the space

$$
D_{\xi}=\left\{\xi x+H_{0}^{1}\left(\Omega ; \mathbb{R}^{n}\right)\right\} .
$$

Instead of considering a functional of the gradient $D u$, we now look at a functional of the symmetrized gradient, i.e. the strain tensor $e(u)=\frac{1}{2}\left(D u+D u^{t}\right)$. We consider the following minimization problem

$$
\inf _{u \in D_{\xi}} F(u):=\int_{\Omega} f(e(u)) d x
$$

where the integrand $f$ is a function from $\mathbb{R}^{n^{2}}$ into $\mathbb{R}^{+}$, defined by

$$
f(\eta)=\min (\lambda+A \eta \cdot \eta, B \eta \cdot \eta)
$$


where $\lambda>0$ and $A, B$ are two positive definite fourth-order tensors defined by

$$
\begin{aligned}
& A \eta=2 \mu_{A} \eta+\left(\kappa_{A}-\frac{2 \mu_{A}}{n}\right) \operatorname{tr}(\eta) I_{2}, \\
& B \eta=2 \mu_{B} \eta+\left(\kappa_{B}-\frac{2 \mu_{B}}{n}\right) \operatorname{tr}(\eta) I_{2} .
\end{aligned}
$$

The positive constants $\kappa$ and $\mu$ are the bulk and shear moduli respectively. The two tensors are assumed to be well-ordered, i.e. $B \eta \cdot \eta \geq A \eta \cdot \eta$ for any $\eta$, or equivalently

$$
\mu_{A} \leq \mu_{B} \text { and } \kappa_{A} \leq \kappa_{B}
$$

As in the conductivity case, the functional $F$ is not quasiconvex, and the minimization problem (47-48) is generically ill-posed. Rather, one needs to introduce the relaxed functional

$$
\bar{F}(u)=\int_{\Omega} Q f(e(u)) d x,
$$

where $Q f$ is the quasiconvexification of $f$, defined by

$$
Q f(\eta)=\inf _{\phi \in H_{0}^{1}\left(Y, \mathbb{R}^{n}\right)} \int_{Y} f(\eta+e(\phi)) d y .
$$

An analogous result to Lemma 3.1 holds (its proof is identical, so we omit it).

Lemma 5.1 The quasiconvexification of $f$, defined by (48), is

$$
Q f(\eta)=\inf _{0 \leq \theta \leq 1} \inf _{A^{*} \in G_{\theta}}\left\{A^{*} \eta \cdot \eta+\lambda \theta\right\}
$$

where $G_{\theta}$ is the set of all symmetric fourth-order tensors which are obtained by periodic homogenization of a mixture of $A$ and $B$ in proportions $\theta$ and $1-\theta$.

In the present setting of elasticity, the set $G_{\theta}$ is unknown. However, it is still possible to compute the so-called Hashin-Shtrikman bound $f(\theta, \eta)$ which is the minimal value of the energy $A^{*} \eta \cdot \eta$ when $A^{*}$ runs into $G_{\theta}$. We recall the result of this computation (see e.g. [2], [3], [13], [21]).

Proposition 5.2 Assume the Poisson ratio of $A$ is positive, i.e. $\lambda_{A}=\kappa_{A}-$ $2 \mu_{A} / n \geq 0$ or the space dimension is $n=2$. Let $\eta_{1} \leq \cdots \leq \eta_{n}$ be the eigenvalues of the symmetric matrix $\eta$. The quasiconvexification of $f$, defined by (48), is

$$
Q f(\eta)=\min _{0 \leq \theta \leq 1}\{f(\theta, \eta)+\lambda \theta\}
$$

where $f(\theta, \eta)$ is a strictly convex function of $\theta$ given by

$$
f(\theta, \eta)=A \eta \cdot \eta+(1-\theta) \max _{\tau}\left(2 \eta \cdot \tau-(B-A)^{-1} \tau \cdot \tau-\theta g(\tau)\right),
$$


where

$$
g(\tau)= \begin{cases}\frac{\left(\tau_{1}-\tau_{n}\right)^{2}}{4 \mu_{A}}+\frac{\left(\tau_{1}+\tau_{n}\right)^{2}}{4\left(\lambda_{A}+\mu_{A}\right)} & \text { if } \tau_{n} \geq \frac{2 \mu_{A}+\lambda_{A}}{2\left(\mu_{A}+\lambda_{A}\right)}\left(\tau_{1}+\tau_{n}\right) \geq \tau_{1}, \\ \frac{\tau_{1}^{2}}{2 \mu_{A}+\lambda_{A}} & \text { if } \tau_{1}>\frac{2 \mu_{A}+\lambda_{A}}{2\left(\mu_{A}+\lambda_{A}\right)}\left(\tau_{1}+\tau_{n}\right), \\ \frac{\tau_{n}^{2}}{2 \mu_{A}+\lambda_{A}} & \text { if } \tau_{n}<\frac{2 \mu_{A}+\lambda_{A}}{2\left(\mu_{A}+\lambda_{A}\right)}\left(\tau_{1}+\tau_{n}\right),\end{cases}
$$

where $\tau_{1} \leq \cdots \leq \tau_{n}$ are the eigenvalues of the symmetric matrix $\tau$.

The assumption $\lambda_{A}=\kappa_{A}-2 \mu_{A} / n \geq 0$ is used only for obtaining a convenient formula for $g(\tau)$ (see [2] for details). In (51) the maximization in $\tau$ is that of a strictly concave function of $\tau$. Therefore, there exists a unique maximizer $\tau$. This allows to prove easily that $f(\theta, \eta)$ is strictly convex in $\theta$. The convexity and differentiability properties of $f(\theta, \eta)$ have been studied in [14] for two anisotropic well-ordered elastic phases. The maximization in $\tau$ can be performed explicitly in dimension $n=2$ (see e.g. [2], [11], [13]), but the explicit minimization in $\theta$ seems to be a formidable task. However, when $B=+\infty$, the algebra is much simpler, and the quasiconvexification can be explicitly computed.

Theorem 5.3 Assume that $\mu_{B}=\kappa_{B}=+\infty$ and that the Poisson ratio of $A$ is positive, i.e. $\lambda_{A}=\kappa_{A}-2 \mu_{A} / n \geq 0$. Let $\eta_{1} \leq \cdots \leq \eta_{n}$ be the eigenvalues of the symmetric matrix $\eta$. Define

$$
g^{*}(\eta)=\mu_{A}\left(\sum_{i=1}^{n}\left|\eta_{i}\right|\right)^{2}+\left(\mu_{A}+\lambda_{A}\right)\left(\sum_{i=1}^{n} \eta_{i}\right)^{2} .
$$

The quasiconvexification of $f$, defined by (48), is

$$
Q f(\eta)= \begin{cases}A \eta \cdot \eta+\lambda & \text { if } g^{*}(\eta) \geq \lambda, \\ A \eta \cdot \eta+2 \sqrt{\lambda g^{*}(\eta)}-g^{*}(\eta) & \text { if } g^{*}(\eta)<\lambda .\end{cases}
$$

We now return to the case $B<+\infty$. As in the conductivity case, the rank-one convexification and the quasiconvexification of $f$ coincide.

Proposition 5.4 The rank-one convex and quasiconvex envelopes of $f$, defined by (48), are equal, i.e. $Q f=R f$.

Remark 5.5 The key ingredient of Proposition 5.4 is the fact that the HashinShtrikman bound $f(\theta, \eta)$ is achieved by sequential laminates. Then, we establish a connection between the lamination formula and the so-called Kohn-Strang algorithm for computing $R f$. This is indeed a very general connection which has also been remarked independently by Milton (see the last chapter in [22]).

Theorem 5.6 Let $\xi_{1} \leq \cdots \leq \xi_{n}$ be the eigenvalues of the symmetric matrix $\xi$. The minimization problem (47-48) has a minimizer over $D_{\xi}$ if and only if, 
either $Q f(\xi)=f(\xi)$, or $Q f(\xi)<f(\xi)$ and $\xi$ or $-\xi$ is a positive definite matrix such that its eigenvalues $\left(\xi_{i}\right)_{1 \leq i \leq n}$ satisfy

$$
\frac{\xi_{i}}{\operatorname{tr}(\xi)} \geq \frac{2 \mu_{A}+\lambda_{A}}{n\left(2 \mu_{A}+\lambda_{A}+\theta\left(\kappa_{B}-\kappa_{A}\right)\right)} .
$$

Remark 5.7 The existence part of Theorem 5.6 is actually proved in [16] in 2-D, and in [15] in higher dimensions. When $B=+\infty$, Theorem 5.6 is still valid and the condition (55) of existence simplifies in $\xi$ or $-\xi$ being a positive definite matrix. The condition on $\xi$ for having $Q f(\xi)=f(\xi)$ is not explicit (it is equivalent to the optimal $\theta$ in (50) being equal to 0 or 1). However, when

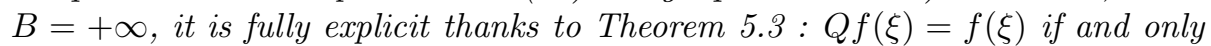
if, either $\xi=0$, or $g^{*}(\xi) \geq \lambda$.

Remark 5.8 When $B=+\infty$, Theorem 5.6 holds true without any technical hypothesis as in [1]. This is due to the fact that our proof relies on the HashinShtrikman variational principle rather than on the explicit knowledge of $G_{\theta}$. Such a proof is also correct in the conductivity case, and therefore allows to improve the result of [1] by removing any additional hypothesis for the nonexistence case (cf. Theorem 1.1).

Proof of Proposition 5.2. We have to prove that $f(\theta, \eta)$ defined by

$$
f(\theta, \eta)=\inf _{A^{*} \in G_{\theta}} A^{*} \eta \cdot \eta,
$$

is also equal to

$$
A \eta \cdot \eta+(1-\theta) \max _{\tau}\left(2 \eta \cdot \tau-(B-A)^{-1} \tau \cdot \tau-\theta g(\tau)\right),
$$

where $g(\tau)$ is defined by (52). This equality is classical and is obtained by using the well-known Hashin-Shtrikman principle (see, for example, [2], [21]). We briefly sketch its proof since we shall rely upon it in the proof of Theorem 5.6.

We start from the formula defining $A^{*}$ obtained by periodic homogenization

$$
A^{*} \eta \cdot \eta=\min _{\phi \in H_{\#}^{1}\left(Y, \mathbb{R}^{n}\right)} \int_{Y}(\chi(y) A+(1-\chi(y)) B)(\eta+e(\phi)) \cdot(\eta+e(\phi)) d y .
$$

By adding and subtracting a reference energy $A(\eta+e(\phi)) \cdot(\eta+e(\phi))$, we obtain

$$
\begin{gathered}
A^{*} \eta \cdot \eta=\min _{\phi}\left[\int_{Y}(1-\chi(y))(B-A)(\eta+e(\phi)) \cdot(\eta+e(\phi)) d y+\right. \\
\left.\int_{Y} A(\eta+e(\phi)) \cdot(\eta+e(\phi)) d y\right] .
\end{gathered}
$$


Using the positivity of $B-A$ and convex duality, the first integral in the right hand side is rewritten

$$
\sup _{\tau(y)} \int_{Y}(1-\chi(y))\left(2 \tau(y) \cdot(\eta+e(\phi)(y))-(B-A)^{-1} \tau(y) \cdot \tau(y)\right) d y .
$$

One can get a lower bound in (56) by specializing to constant tensors $\tau$

$$
\geq 2(1-\theta) \tau \cdot \eta-(1-\theta)(B-A)^{-1} \tau \cdot \tau+\int_{Y} 2(1-\chi(y)) \tau \cdot e(\phi)(y) d y,
$$

where $\theta=\int_{Y} \chi(y) d y$. After some simplification, this yields

$$
\begin{aligned}
A^{*} \eta \cdot \eta \geq & A \eta \cdot \eta+2(1-\theta) \eta \cdot \tau-(1-\theta)(B-A)^{-1} \tau \cdot \tau+ \\
& \min _{\phi(y)} \int_{Y}[A e(\phi) \cdot e(\phi)+2(1-\chi(y)) \tau \cdot e(\phi)] d y .
\end{aligned}
$$

The above infimum in $\phi$ is easily computed by Fourier analysis (see e.g. [2]). Denoting by $\hat{\chi}(k)$ the Fourier component at frequency $k$ of the characteristic function $\chi(y)$, it is exactly equal to

$$
-\sum_{k \neq 0}|\hat{\chi}(k)|^{2} g(\tau, k)
$$

where

$$
g(\tau, k)=\frac{1}{\mu_{A}}\left(|\tau k|^{2}-(\tau k \cdot k)^{2}\right)+\frac{1}{2 \mu_{A}+\lambda_{A}}(\tau k \cdot k)^{2} .
$$

Optimizing over $k$ gives when $\lambda_{A}>0$ (see Proposition 7.4 in [2])

$$
g(\tau)=\sup _{k \neq 0} g(\tau, k)
$$

Remarking that

$$
\sum_{k \neq 0}|\hat{\chi}(k)|^{2}=\theta(1-\theta)
$$

the quantity (59) is bounded from below by $-\theta(1-\theta) g(\tau)$. Varying $\tau$ among all constant symmetric matrices gives the lower bound

$$
A^{*} \eta \cdot \eta \geq A \eta \cdot \eta+(1-\theta) \max _{\tau}\left(2 \eta \cdot \tau-(B-A)^{-1} \tau \cdot \tau-\theta g(\tau)\right) .
$$

To conclude the proof it remains to prove its attainability. This is a classical matter by using the layering formula (see (61) below). For details, the interested reader is referred to $[2],[21]$.

Proof of Theorem 5.3. When $B=+\infty$, we deduce from Proposition 5.2 that

$$
f(\theta, \eta)=A \eta \cdot \eta+\frac{(1-\theta)}{\theta} g^{*}(\eta)
$$


where $g^{*}(\eta)$ is the convex conjugate of $g(\tau)$. A simple minimization with respect to $\theta$ gives the values of $Q f(\eta)$. To compute $g^{*}(\eta)=\max _{\tau}(2 \tau \cdot \eta-g(\tau))$, we assume with no loss of generality that $\eta$ has at least a positive eigenvalue, i.e. $\eta_{n}>0$. If all the eigenvalues of $\eta$ are positive, i.e. $0<\eta_{1} \leq \ldots \leq \eta_{n}$, then it is easily seen that the unique maximizer $\tau$ is equal to $\left(2 \mu_{A}+\lambda_{A}\right) \operatorname{tr}(\eta) I_{2}$, which gives the desired value (53). If not, we introduce the integer $p \in\{1, \ldots, n-1\}$ such that $\eta_{1} \leq \ldots \leq \eta_{p} \leq 0<\eta_{p+1} \leq \ldots \leq \eta_{n}$. In this case, let us show that there exists an optimal $\tau$ with eigenvalues $\tau_{1} \leq \cdots \leq \tau_{n}$ which lies in the first regime of $(52)$, i.e.

$$
g(\tau)=\frac{\left(\tau_{1}-\tau_{n}\right)^{2}}{4 \mu_{A}}+\frac{\left(\tau_{1}+\tau_{n}\right)^{2}}{4\left(\lambda_{A}+\mu_{A}\right)} .
$$

If all eigenvalues $\eta_{i}$ are non-zero, the optimality condition delivers the unique maximizer $\tau$ given by

$$
\begin{gathered}
\tau_{1}=\ldots=\tau_{p}=\left(2 \mu_{A}+\lambda_{A}\right) \sum_{i=1}^{p} \eta_{i}+\lambda_{A} \sum_{i=p+1}^{n} \eta_{i}, \\
\tau_{p+1}=\ldots=\tau_{n}=\lambda_{A} \sum_{i=1}^{p} \eta_{i}+\left(2 \mu_{A}+\lambda_{A}\right) \sum_{i=p+1}^{n} \eta_{i},
\end{gathered}
$$

and the maximal value is exactly (53). One easily check that $\tau_{1}<\tau_{n}$ and

$$
\tau_{n} \geq \frac{2 \mu_{A}+\lambda_{A}}{2\left(\mu_{A}+\lambda_{A}\right)}\left(\tau_{1}+\tau_{n}\right) \geq \tau_{1},
$$

which implies that $\tau$ is indeed optimal in the definition of $g^{*}(\eta)$. When one eigenvalue $\eta_{i}$ is equal to zero, the optimal $\tau$ is not unique but the above formula still define one such optimal $\tau$ which is not a scalar matrix.

Remark that a necessary and sufficient condition for the existence of a maximizer $\tau$, in the definition of $g^{*}(\eta)$, which is not proportional to the identity $I_{2}$ is that $\eta$ has one zero eigenvalue or two eigenvalues of opposite signs.

Proof of Proposition 5.4. The proof is almost identical to that of Proposition 3.5. Let us notice first that, in the elasticity case, a function $g$ is said to be rankone convex if it is rank-one convex in the space $\mathbb{R}_{s}^{n \times n}$ of symmetric matrices, i.e.

$$
g(t \sigma+(1-t) \tau) \leq t g(\sigma)+(1-t) g(\tau),
$$

for all $t \in[0,1]$, and for all symmetric matrices $\tau, \sigma$ such that rank $(\tau-\sigma) \leq 1$.

The lamination formula is also slightly different in elasticity (cf. [12]). A rank- $p$ sequential laminate $A_{p} \in L_{\theta}$, obtained by mixing $A$ and $B$ in proportion $\theta$ 
and $(1-\theta)$ respectively, is determined by the lamination directions $\left(e_{i}\right)_{1 \leq i \leq p}$ and the lamination parameters $\left(m_{i}\right)_{1 \leq i \leq p}$, satisfying $0 \leq m_{i} \leq 1$ and $\sum_{i=1}^{p} m_{i}=1$,

$$
(1-\theta)\left(A_{p}-A\right)^{-1}=(B-A)^{-1}+\theta \sum_{i=1}^{p} m_{i} f_{A}\left(e_{i}\right),
$$

where $f_{A}\left(e_{i}\right)$ is a positive non-definite fourth-order tensor defined by the quadratic form

$$
f_{A}(e) \xi \cdot \xi=\frac{1}{\mu_{A}}\left(|\xi e|^{2}-(\xi e \cdot e)^{2}\right)+\frac{1}{2 \mu_{A}+\lambda_{A}}(\xi e \cdot e)^{2} .
$$

Remark that in (61) it is always possible to take $p$ bounded by application of Caratheodory theorem since the set of all fourth order tensors $\sum_{i=1}^{p} m_{i} f_{A}\left(e_{i}\right)$ is convex. Moreover, a generalization of Lemma 4.2 states that the HashinShtrikman bound (50) is indeed attained by a sequential laminate of rank at most $n$ (see e.g. [2]). Therefore, as in the conductivity case, only $n$ steps of the Kohn-Strabg algorithm are necessary to reach the rank-one convex envelope $R f$.

The only modifications in the proof consist in using the eigenvalues and the eigenvectors $\left(e_{k}\right)_{1 \leq k \leq n}$ of the symmetric matrix $\eta$ instead of its singular values and directions. Consequently, the formula (41) can be written as follows

$$
c_{k}^{*}=\frac{\left(A_{k}-A\right) \tilde{\eta} \cdot E_{k+1}}{\left(2 \mu_{A}+\lambda_{A}\right)\left(1-\theta_{k+1}\right)+\theta_{k+1} A_{k} E_{k+1} \cdot E_{k+1}},
$$

with $E_{k+1}=e_{k+1} \otimes e_{k+1}$. The end of the proof can be reproduced step by step by noticing that

$$
f_{A}\left(e_{k+1}\right) \xi_{k} \cdot \xi_{k}=\frac{1}{2 \mu_{A}+\lambda_{A}}\left(\xi_{k} e_{k+1} \cdot e_{k+1}\right)^{2}
$$

with $\xi_{k}=\left(A_{k}-A\right) \tilde{\eta}^{\prime}$. Let us emphasize that we can not obtain easily an explicit formula for the tensors $A_{k}$, although it was possible in the conductivity case.

Proof of Theorem 5.6. When $Q f(\xi)=f(\xi)$, it is easy to check that $u(x)=\xi x$ is a minimizer in $D_{\xi}$ for (47-48). In the remaining case $Q f(\xi)<f(\xi)$, the existence or not of a minimizer will be governed by the nature of the unique optimal $\tau$ in (51). We shall prove that there exists a minimizer if and only if this optimal $\tau$ is proportional to the identity $I_{2}$. Such a minimizer will be obtained with the confocal ellipsoids construction which has been studied in the context of elasticity by Grabovsky and Kohn (see [16], or section 6 in [15]).

In a first step, we prove by contradiction that, if the optimal $\tau$ in (51) is not a scalar matrix, then there is no minimizer of problem (47-48). As in Theorem 3.9 , we assume that there exists a minimizer $u(x) \in D_{\xi}$, and we denote by $Z_{u}$ the following subset of $\Omega$

$$
Z_{u}=\{x \in \Omega, B e(u) \cdot e(u) \leq A e(u) \cdot e(u)+\lambda\} .
$$


By Vitali's theorem, we cover the unit cube $Y=[0,1]^{n}$ by a countable family $\left(\Omega_{i}\right)_{i \geq 1}$ of disjoint homothetics of $\Omega$. We also denote by $\left(Z_{u i}\right)_{i \geq 1}$ the family of the homothetics of $Z_{u}$ in $\Omega_{i}$. Let $\chi(x)$ and $\tilde{\chi}(y)$ be the characteristic functions respectively of $\Omega \backslash Z_{u}$ and $Y \backslash \cup_{i \geq 1} Z_{u i}$.

We introduce the homogenized tensor $A^{*}$ associated to $\chi$ defined by

$$
A^{*} \xi \cdot \xi=\min _{\phi \in H_{\#}^{1}\left(Y ; \mathbb{R}^{n}\right)} \int_{Y}(\tilde{\chi} A+(1-\tilde{\chi}) B)(\xi+e(\phi)) \cdot(\xi+e(\phi)) d y .
$$

We are going to show that the characteristic function $\tilde{\chi}$ is invariant in one direction by using the Hashin-Shtrikman variational principle for the tensor $A^{*}$ at the energy $\xi$. If this is true, namely if, in some basis, $\frac{\partial \tilde{\chi}}{\partial y_{n}}(y)=0$ in $Y$, then the same trick used at the end of the proof of Theorem 3.9 (using a special choice of Vitali covering with two identical copies of $\Omega$ slightly misfitted) leads to a contradiction.

From Proposition 5.2 we know that

$$
A^{*} \xi \cdot \xi \geq f\left(\theta_{u}, \xi\right)
$$

where $f\left(\theta_{u}, \xi\right)$ is the value of the Hashin-Shtrikman bound with $\theta_{u}=\frac{1}{|\Omega|} \int_{\Omega} \chi(x) d x$.

However, using again in (62) the test function obtained by pasting together the properly rescaled copies of $u(x)-\xi x$ in each $\Omega_{i}$ gives

$$
A^{*} \xi \cdot \xi \leq \frac{1}{|\Omega|} \int_{\Omega}(\chi A+(1-\chi) B) e(u) \cdot e(u) d x=Q f(\xi)-\lambda \theta_{u}
$$

since $u$ is a minimizer. The same argument as in Theorem 3.9 leads to

$$
A^{*} \xi \cdot \xi=f\left(\theta_{u}, \xi\right) \text {. }
$$

Therefore, in view of (64) $A^{*}$ achieves equality in the Hashin-Shtrikman bound. Recall from the proof of Proposition 5.2 that the Hashin-Shtrikman bound was established by making two inequalities in (56) and (59). For the present tensor $A^{*}$ these inequalities must be actually equalities. Let $\tau$ be the non-scalar matrix which is optimal in (51). We focus on (59) which is now the following equality

$$
\sum_{k \neq 0}|\hat{\chi}(k)|^{2} g(\tau, k)=\theta(1-\theta) g(\tau)
$$

where $g(\tau)=\sup _{k \neq 0} g(\tau, k)$ and

$$
g(\tau, k)=\frac{1}{\mu_{A}}\left(|\tau k|^{2}-(\tau k \cdot k)^{2}\right)+\frac{1}{2 \mu_{A}+\lambda_{A}}(\tau k \cdot k)^{2} .
$$

If $\tau$ is proportional to the identity, then $g(\tau, k)$ is constant, equal to $g(\tau)$ for any $k$, and (65) is an obvious equality. If $\tau$ is not proportional to the identity, 
then $g(\tau, k)$ is obviously not constant. However, in view of $(65) g(\tau, k)$ must be equal to $g(\tau)$ for any $k$ such that $\hat{\chi}(k) \neq 0$. Therefore, for any $k$ such that $g(\tau, k)<g(\tau)$, we necessarily have $\hat{\chi}(k)=0$. This implies that $\chi(y)$ does not depend on the component $y \cdot k$, and leads to the desired contradiction.

In a second step, we find a necessary condition on $\xi$ for the optimal $\tau$ to be a scalar matrix. We write the optimality condition of (51) (for details, see [2])

$$
0 \in 2 \xi-2(B-A)^{-1} \tau-\theta \partial g(\tau)
$$

where $\partial g(\tau)$ is the subdifferential of $g(\tau)$. Since $g(\tau)$ is the supremum of quadratic functions (cf. (60)), its subdifferential is the convex hull of the gradients of those quadratic functions which are extremal in (60). Specifying (66) for $\tau=t I_{2}$, with $t \in \mathbb{R}$, yields

$$
\xi=\frac{t}{n\left(\kappa_{B}-\kappa_{A}\right)} I_{2}+\frac{\theta}{2 \mu_{A}+\lambda_{A}} t \sum_{i=1}^{p} m_{i} e_{i} \otimes e_{i}
$$

where $\left(e_{i}\right)_{1 \leq i \leq p}$ are unit vectors and the parameters $\left(m_{i}\right)_{1 \leq i \leq p}$ satisfy $m_{i} \geq 0$ and $\sum_{i=1}^{p} m_{i}=1$. Taking the trace of (67) yields the value of $t$

$$
\left(\frac{1}{\left(\kappa_{B}-\kappa_{A}\right)}+\frac{\theta}{2 \mu_{A}+\lambda_{A}}\right) \frac{\xi}{\operatorname{tr}(\xi)}=\frac{1}{n\left(\kappa_{B}-\kappa_{A}\right)} I_{2}+\frac{\theta}{2 \mu_{A}+\lambda_{A}} \sum_{i=1}^{p} m_{i} e_{i} \otimes e_{i}
$$

Since the sum $\sum_{i=1}^{p} m_{i} e_{i} \otimes e_{i}$ is positive, it implies that $\xi$ or $-\xi$ is positive definite and each of its eigenvalue satisfy

$$
\left(\frac{1}{\left(\kappa_{B}-\kappa_{A}\right)}+\frac{\theta}{2 \mu_{A}+\lambda_{A}}\right) \frac{\xi_{i}}{\operatorname{tr}(\xi)} \geq \frac{1}{n\left(\kappa_{B}-\kappa_{A}\right)},
$$

which is nothing but condition (55). Therfore, (55) is a necessary condition for the optimal $\tau$ to be a scalar matrix. It turns out that (55) is precisely condition (6.11) in [15] for the existence of the confocal ellipsoid construction optimal for $\xi$. This concludes the proof.

Remark 5.9 We briefly explain why the proof of Theorem 5.6 is still valid when $B=+\infty$. We know from Theorem 5.3 that if $\xi$ or $-\xi$ is positive definite, then there exists a unique optimal $\tau$ in (51) being proportional to the identity $I_{2}$. In this case, the confocal ellipsoids construction of Grabovsky still yields a minimizer. If $\xi$ has one zero eigenvalue or eigenvalues of opposite signs, then there is an optimal $\tau$ (possibly non-unique) which is not a scalar matrix. The contradiction argument is still valid by defining the homogenized tensor $A^{*}$ as follows to $\chi$ defined by

$$
A^{*} \xi \cdot \xi=\inf _{\phi \in H_{\#}^{1}\left(Y ; \mathbb{R}^{n}\right)} \int_{Y} \tilde{\chi}(y) A(\xi+e(\phi)) \cdot(\xi+e(\phi)) d y
$$


with $\xi+e(\phi)(y)=0$ whenever $\tilde{\chi}(y)=0$ in $Y$. Remark that (68) makes sense (i.e. $\left.A^{*} \xi \cdot \xi<+\infty\right)$ since there exists at least one test function satisfying the constraint which is obtained from $u(x)-\xi x$. Eventually, the Hashin-Shtrikman bound is also valid when $B=+\infty$ (cf. the proof of Proposition 5.2).

Acknowledgements. Part of this work was carried out while the first author was a researcher at the French Atomic Energy Commission (CEA Saclay, France), the support of which is kindly acknowledged. We thank Gilles Francfort and Graeme Milton for stimulating comments and remarks on this work.

\section{References}

[1] ALLAIRE G., FRANCFORT G.A., Existence of minimizers for nonquasiconvex functionals arising in optimal design, Ann. Inst. H. Poincaré, $15,(1998)$.

[2] ALLAIRE G., KOHN R.V., Optimal bounds on the effective behavior of a mixture of two well-ordered elastic materials, Quat. Appl. Math. 51, 643674 (1993).

[3] AVELLANEDA M., Optimal bounds and microgeometries for elastic twophase composites, SIAM J. Appl. Math. 47, 6, 1216-1228 (1987).

[4] BALL J.M., JAMES R.D., Fine phase mixtures as minimizers of energy, Arch. Rat. Mech. Anal. 100, 1, 13-52 (1987).

[5] BALL J.M., MURAT F., $W^{1, p}$ quasiconvexity and variational problems for multiple integrals, J. Func. Anal. 58, 225-253 (1984).

[6] BEnSOUSSAN A., LIONS J.L., PAPANICOLAOU G., Asymptotic analysis for periodic structures, North-Holland, Amsterdam (1978).

[7] DACOROGNA B., Direct Methods in the Calculus of Variations, Springer Verlag, Berlin Heidelberg (1989).

[8] DACOROGNA B., MARCELLINI P., Existence of minimizers for non quasiconvex integrals, Arch. Rational Mech. Anal. 131, 359-399 (1995).

[9] DAL MASO G., KOHN R.V., The local character of $G$-closure, to appear.

[10] FIROOZYE N., KOHN R.V., Geometric parameters and the relaxation of multiwell energies, in Microstructure and phase transitions, D. Kinderlehrer et al. eds., pp.85-109, Springer Verlag, New York (1993). 
[11] FRANCFORT G., MARIGO J.-J., Stable damage evolution in a brittle continuous medium, Eur. J. Mech. A/Solids 12, pp.149-189 (1993).

[12] FRANCFORT G., MURAT F., Homogenization and Optimal Bounds in Linear Elasticity, Arch. Rat. Mech. Anal., 94, 307-334 (1986).

[13] GIBIANSKI L., CHERKAEV A., Microstructures of composites of extremal rigidity and exact bounds of the associated energy density, in Topics in the mathematical modeling of composite materials, A. Cherkaev and R.V. Kohn eds., series : Progress in Nonlinear Differential Equations and their Applications, pp.273-317, Birkhaüser, Boston (1997), russian version: Ioffe Physicotechnical Institute preprint (1987).

[14] GRABOVSKY Y., Nonsmooth analysis and quasi-convexification in elastic energy minimization problems, Structural Optimization 10, pp.217-221 (1995).

[15] GRABOVSKY Y., Bounds and extremal microstructures for twocomponent composites: a unified treatment based on the translation method, Proc. Roy. Soc. London Series A 452, pp.919-944 (1996).

[16] GRABOVSKY Y., KOHN R.V., Microstructures minimizing the energy of a two-phase elastic composite in two space dimensions I: the confocal ellipse construction, J. Mech. Phys. Solids 43, pp.933-947 (1995).

[17] KOHN R.V., Relaxation of a double-well energy, Cont. Mech. and Thermodyn., 3, pp.193-236 (1991).

[18] KOHN R.V., STRANG G., Optimal design and relaxation of variational problems I, II, III, Comm. Pure and Appl. Math. 39, 113-182, 353-377 (1986).

[19] LURIE K., CHERKAEV A., Exact estimates of conductivity of composites formed by two isotropically conducting media, taken in prescribed proportion, Proc. Royal Soc. Edinburgh 99A, pp.71-87 (1984).

[20] LURIE K., CHERKAEV A., Exact estimates of the conductivity of a binary mixture of isotropic materials, Proc. Royal Soc. Edinburgh 104A, pp.21-38 (1986).

[21] MILTON G., On characterizing the set of possible effective tensors of composites: the variational method and the translation method, Comm. Pure Appl. Math., XLIII, pp.63-125 (1990).

[22] MILTON G., Effective tensors of composites, book to appear.

[23] MIRSKY L., On the trace of a matrix product, Math. Nachr. 20, 171-174 (1959). 
[24] MURAT F., Contre-exemples pour divers problèmes où le contrôle intervient dans les coefficients, Ann. Mat. Pura Appl. 112, 49-68 (1977).

[25] MURAT F., TARTAR L., $H$-convergence, in Topics in the mathematical modeling of composite materials, A. Cherkaev and R.V. Kohn eds., series : Progress in Nonlinear Differential Equations and their Applications, pp.21-44, Birkhaüser, Boston (1997), french version: mimeographed notes, séminaire d'Analyse Fonctionnelle et Numérique de l'Université d'Alger (1978).

[26] MURAT F., TARTAR L., Calculus of variations and homogenization, in Topics in the mathematical modeling of composite materials, A. Cherkaev and R.V. Kohn eds., series : Progress in Nonlinear Differential Equations and their Applications, pp.139-173, Birkhaüser, Boston (1997), french version: Calcul des Variations et Homogénéisation, in Les Méthodes de l'Homogénéisation Théorie et Applications en Physique, Coll. Dir. Etudes et Recherches EDF, Eyrolles, 319-369 (1985).

[27] TARTAR L., Estimations fines de coefficients homogénéisés, Ennio de Giorgi Colloquium, P. Krée ed., Pitman Research Notes in Math. 125, 168-187 (1985). 